\section{Butterflies (Lepidoptera) of Dibang Valley, Mishmi Hills, Arunachal Pradesh, India}

\section{Monsoon Jyoti Gogoi}

Centre for Biodiversity and Natural Resource Conservation, Department of Ecology and Environmental Science, Assam University, Silchar, Assam 788011, India

Email: monsoonjyoti@gmail.com

The Mishmi Hills $\left(95^{\circ} 49^{\prime}-95^{\circ} 59^{\prime}\right.$ N \& 28 $06^{\prime}-$ $28^{\circ} 23^{\prime} \mathrm{E}$ ) are located in the eastern region of Arunachal Pradesh. The altitude varies from 400-3568 m. The hill range lies to the north of the Assam Valley.

The major part of the Mishmi Hills is in the Dibang Valley districts of Arunachal Pradesh. The Dibang Valley districts have two divisions - Lower and Upper Dibang Valley. The headquarters of the two districts are Roing and Anini, respectively. Roing lies on the plains near the Lohit River while Anini is at an elevation in the mountains and near the international border with China. The valley is bounded on the north by China, on the east by Lohit District of Arunachal Pradesh, on the west by Siang District of Arunachal Pradesh and on the south by Tinsukia District of Assam. Part of the Lower Dibang Valley $\left(281.5 \mathrm{~km}^{2}\right)$

Date of publication (online): 10 October 2012

Date of publication (print): 10 October 2012

ISSN 0974-7907 (online) | 0974-7893 (print)

Editor: Peter Smetacek

Manuscript details:

Ms \# 02975

Received 25 October 2011

Final received 14 September 2012

Finally accepted 16 September 2012

Citation: Gogoi, M.J. (2012). Butterflies (Lepidoptera) of Dibang Valley, Mishmi Hills, Arunachal Pradesh, India. Journal of Threatened Taxa 4(12): 3137-3160.

Copyright: () Monsoon Jyoti Gogoi 2012. Creative Commons Attribution 3.0 Unported License. JoTT allows unrestricted use of this article in any medium for non-profit purposes, reproduction and distribution by providing adequate credit to the authors and the source of publication.

Acknowledgements: The work was undertaken by the author under North Orissa University, to complete fourth semester M.Sc. dissertation in Wildlife and Conservation Biology. I am thankful to Ipra Mekola, an active wildlife conservationist and respected person of Idu-mishmi community. Along with him, I thank Kavi Angama, Chipra Mekola and Abidur Rahman. I also thank Rakesh Saur who helped in preparing the map.

\section{OPEN ACGESS | FREE DOWNLOAD}

is protected under Mehao Wildlife Sanctuary $\quad\left(93^{0} 30^{\prime}-95^{\circ} 45^{\prime} \mathrm{N} \quad \&\right.$ $\left.28^{\circ} 05^{\prime}-28^{0} 15^{\prime} \mathrm{E}\right)$ (MWS) (Image $1)$.

The Dibang River originates in the western part of China and flows through this valley in a northsouth direction. It is one of the main tributaries of the Brahmaputra.

The vegetation is characterized by tropical evergreen forests (up to $900 \mathrm{~m}$ ), subtropical and temperate forests $(900-1800 \mathrm{~m})$, temperate broad leaf forest $(1800-2800 \mathrm{~m})$ and temperate conifer forest (2800-3500 m) (Champion \& Seth 1968).

\section{Methods}

Study Area: 1. Sally Lake (Image 2): The Lake is about $5 \mathrm{~km}$ from Roing Town and lies at an altitude of about $400 \mathrm{~m}$. The lake is very close to the Deopani River and is surrounded by hills. Small streams flow through the bamboo forest into the open areas. The lake is protected under MWS.

2. Deopani River Bed: The river bed remains dry throughout the year. The river gets its water from the streams originating in the surrounding hills near Deopani. High elevation forest exists in this area. The plains have secondary forest growth.

3. Dibang River-bed (Image 3): It lies west of the Mishmi Hills and is not under the jurisdiction of MSW. There are several small villages like Chidu. The vast grassland of Nizamghat (elevation of 266m) is in this area.

4. Tiwarigaon (Image 4a): A small village on the way to Mayodia, lying at an elevation of around $1300 \mathrm{~m}$. The place is the perfect place for the study of high montane butterflies and is $28 \mathrm{~km}$ from Roing Town.

5. Mayodia pass (Image 4b): The area lies on the way to Anini and is about $56 \mathrm{~km}$ from Roing Town. This area is the highest elevated point on the way from Roing-Hunli at 2,666m and is distinguished by a cold rocky mountain.

\section{Survey methods}

The methodology involved checklist surveys on sunny days from 07 March-22 June, 2011 to determine the maximum number of species. Visual search and 


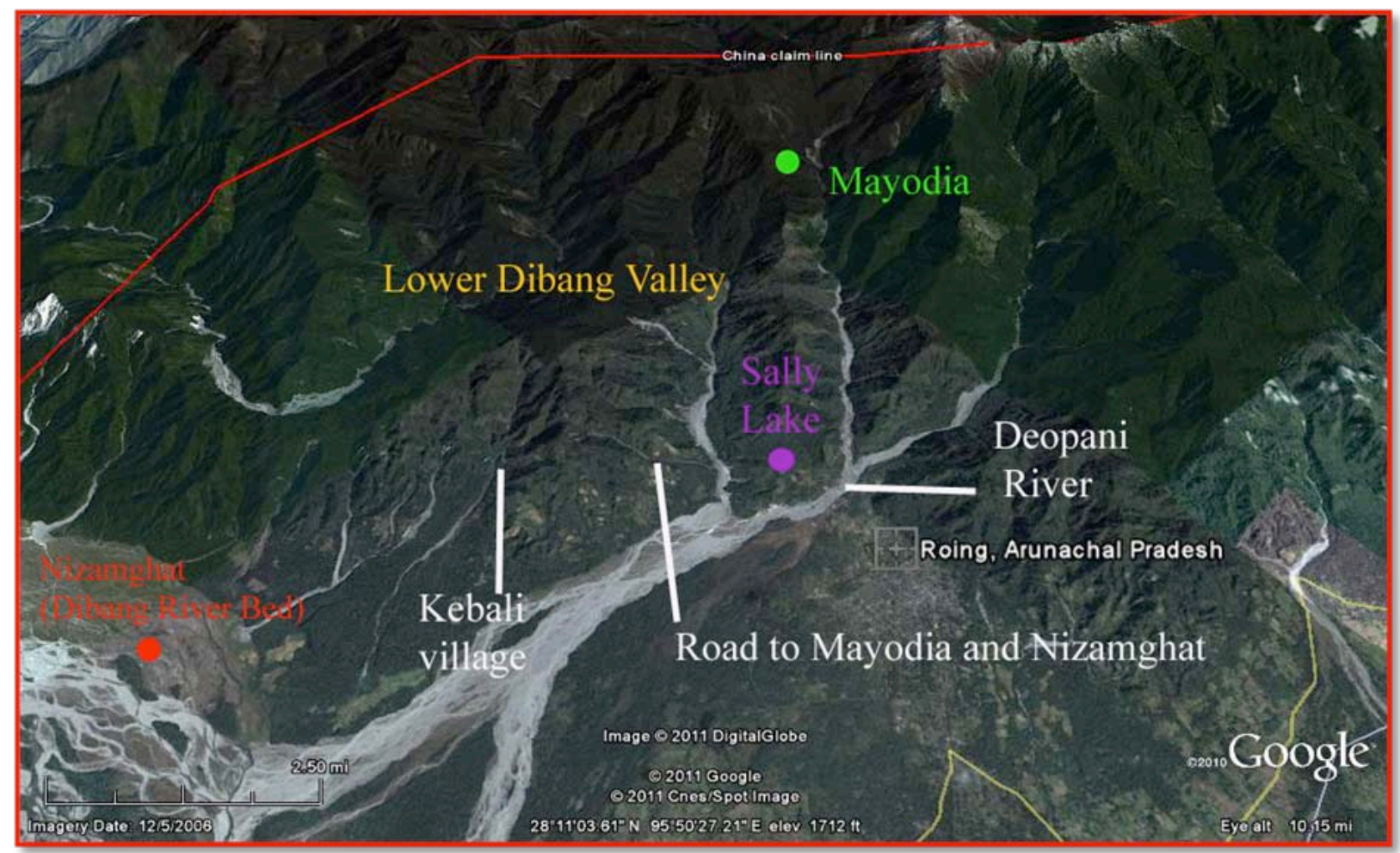

Image 1. Satellite imagery showing areas covered during field study (Map: Rakesh Saur)

photography were conducted on a regular basis during the day. Occasional surveys were undertaken after 16:00hr to locate shade loving butterflies. Searches were conducted near water sources, damp patches in the forest, open sunny areas, blossoming flowers and bird droppings. Searches were also conducted on hill tops, especially in the catchment areas of hill streams, as well as from top to bottom of hill streams to record the maximum number of species. Butterflies were photographed from different angles as often as possible to obtain sufficient photographs to enable positive identification of species. Butterfly species were identified using the identification keys of Watson (1897), Evans (1932), Talbot (1947), Smith (1994), Pinratana (1985), Haribal (1992), and photographic guides of Kehimkar (2008).

\section{Results and Discussion}

The field study resulted in the recording of 294 butterfly species belonging to 156 genera, from Dibang Valley (Table 1). This includes 33 species of Papilionidae, 26 species of Pieridae, 59 species of Lycaenidae, 115 species of Nymphalidae and 61 species belonging to Hesperiidae. Noteworthy rare and endangered species encountered were de Nicéville's Windmill Byasa polla which is included on Schedule I of the Indian Wildlife (Protection) Act (IWPA), (1972); Khaki Silverline Spindasis rukmini is another interesting species which is also included on Schedule I of the IWPA, (1972).

The matter of greatest concern is that Byasa polla, Spindasis rukmini were recorded outside the protected area of MWS. The species were recorded in a small village called Chidu near Nizamghat. The area is undergoing deforestation due to increase of settlements in the area and clearing of land for agriculture. Logging has also been observed on a small scale in the area. The increasing use of pesticides in the citrus orchards is another concern in the area. Overall, the last remaining lowland forest of Lower Dibang Valley is facing a serious threat to its continued existence at present. All these raise serious concerns regarding the survival of butterflies in the area. The record of such rare species in the area shows the potential biodiversity of the area and reflects the need for notification of more protected areas in Arunachal Pradesh.

It is stressed that the present survey covered the period from March-June 2011. Doubtless, there will 
Table 1. Annotated list of butterflies recorded in the Mishmi Hills, Arunachal Pradesh during the study period (March-June 2011).

\begin{tabular}{|c|c|c|}
\hline Scientific name & Common name & $\begin{array}{c}\text { Indian } \\
\text { WPA } 1972\end{array}$ \\
\hline \multicolumn{3}{|l|}{ Papilionidae } \\
\hline $\begin{array}{l}\text { 1. Pachliopta aristolochiae } \\
\text { aristolochiae }\end{array}$ & Common Rose & \\
\hline 2. Troides helena cerberus & Common Birdwing & \\
\hline 3. Troides aeacus aeacus & Golden Birdwing & \\
\hline $\begin{array}{l}\text { 4. Atrophaneura varuna } \\
\text { astorion }\end{array}$ & Common Batwing & \\
\hline 5. Atrophaneura aidoneus & Lesser Batwing & \\
\hline 6. Byasa polla & $\begin{array}{l}\text { De Nicéville's } \\
\text { Windmill }\end{array}$ & $\begin{array}{l}\text { Schedule I } \\
\text { Part IV }\end{array}$ \\
\hline $\begin{array}{l}\text { 7. Byasa polyeuctes } \\
\text { polyeuctes }\end{array}$ & Common Windmill & \\
\hline 8. Byasa dasarada dasarada & Great Windmill & $\begin{array}{l}\text { Schedule II } \\
\text { Part II }\end{array}$ \\
\hline 9. Papilio agestor agestor & Tawny Mime & \\
\hline 10. Papilio epycides epycides & Lesser Mime & \\
\hline 11. Papilio clytia clytia & Common Mime & \\
\hline 12. Papilio polytes romulus & Common Mormon & \\
\hline $\begin{array}{l}\text { 13. Papilio demoleus } \\
\text { demoleus }\end{array}$ & Lime Butterfly & \\
\hline 14. Papilio castor castor & Common Raven & \\
\hline 15. Papilio helenus helenus & Red Helen & \\
\hline 16. Papilio nephelus chaon & Yellow Helen & $\begin{array}{l}\text { Schedule II } \\
\text { Part II }\end{array}$ \\
\hline 17.Papilio memnon agenor & Great Mormon & $\begin{array}{l}\text { Schedule I } \\
\text { Part IV }\end{array}$ \\
\hline $\begin{array}{l}\text { 18. Papilio protenor } \\
\text { euprotenor }\end{array}$ & Spangle & \\
\hline $\begin{array}{l}\text { 19. Papilio alcmenor } \\
\text { alcmenor }\end{array}$ & Redbreast & \\
\hline 20. Papilio polyctor ganesa & Common Peacock & \\
\hline 21. Papilio paris paris & Paris Peacock & \\
\hline $\begin{array}{l}\text { 22. Graphium antiphates } \\
\text { pompilius }\end{array}$ & Fivebar Swordtail & \\
\hline 23. Graphium agetes agetes & Fourbar Swordtail & \\
\hline 24. Graphium doson axion & Common Jay & \\
\hline $\begin{array}{l}\text { 25. Graphium evemon } \\
\text { albociliatis }\end{array}$ & Lesser Jay & $\begin{array}{l}\text { Schedule II } \\
\text { Part II }\end{array}$ \\
\hline $\begin{array}{l}\text { 26. Graphium chironides } \\
\text { chironides }\end{array}$ & Veined Jay & \\
\hline $\begin{array}{l}\text { 27. Graphium agamemnon } \\
\text { agamemnon }\end{array}$ & Tailed Jay & \\
\hline $\begin{array}{l}\text { 28. Graphium sarpedon } \\
\text { sarpedon }\end{array}$ & Common Bluebottle & \\
\hline $\begin{array}{l}\text { 29. Graphium xenocles } \\
\text { xenocles }\end{array}$ & Great Zebra & \\
\hline $\begin{array}{l}\text { 30. Lamproptera curius } \\
\text { curius }\end{array}$ & White Dragontail & \\
\hline $\begin{array}{l}\text { 31. Lamproptera meges } \\
\text { indistincta }\end{array}$ & Green Dragontail & \\
\hline
\end{tabular}

\begin{tabular}{|c|c|c|}
\hline Scientific name & Common name & $\begin{array}{c}\text { Indian } \\
\text { WPA } 1972\end{array}$ \\
\hline $\begin{array}{l}\text { 32. Meandrusa lachinus } \\
\text { lachinus }\end{array}$ & Brown Gorgon & $\begin{array}{l}\text { Schedule } \\
\text { II Part II }\end{array}$ \\
\hline 33. Meandrusa payeni evan & Yellow Gorgon & \\
\hline \multicolumn{3}{|l|}{ Pieridae } \\
\hline $\begin{array}{l}\text { 34. Eurema andersoni } \\
\text { andersoni }\end{array}$ & $\begin{array}{l}\text { One-Spot Grass } \\
\text { Yellow }\end{array}$ & $\begin{array}{l}\text { Schedule II } \\
\text { Part II }\end{array}$ \\
\hline 35. Eurema blanda silhetana & $\begin{array}{l}\text { Three-Spot Grass } \\
\text { Yellow }\end{array}$ & \\
\hline 36. Eurema brigitta rubella & Small Grass Yellow & \\
\hline 37. Eurema hecabe hecabe & $\begin{array}{l}\text { Common Grass } \\
\text { Yellow }\end{array}$ & \\
\hline $\begin{array}{l}\text { 38. Gandaca harina } \\
\text { assamica }\end{array}$ & Tree Yellow & \\
\hline $\begin{array}{l}\text { 39. Dercas verhuelli } \\
\text { doubledayi }\end{array}$ & Tailed Sulphur & \\
\hline $\begin{array}{l}\text { 40. Catopsilia pomona } \\
\text { pomona }\end{array}$ & Common Emigrant & \\
\hline $\begin{array}{l}\text { 41. Catopsilia pyranthe } \\
\text { pyranthe }\end{array}$ & Mottled Emigrant & \\
\hline 42. Colias fieldii fieldii & Dark Clouded Yellow & \\
\hline 43. Ixias pyrene familiaris & Yellow Orange Tip & \\
\hline 44. Pareronia avatar avatar & Pale Wanderer & \\
\hline 45. Appias lyncida hippoides & Chocolate Albatross & \\
\hline 46. Appias nero galba & Orange Albatross & $\begin{array}{l}\text { Schedule } \\
\text { IV }\end{array}$ \\
\hline 47. Appias albina darada & Common Albatross & $\begin{array}{l}\text { Schedule II } \\
\text { Part II }\end{array}$ \\
\hline 48. Appias lalage lalage & Spot Puffin & \\
\hline 49. Appias indra indra & Plain Puffin & $\begin{array}{l}\text { Schedule II } \\
\text { Part II }\end{array}$ \\
\hline 50. Pieris brassicae & $\begin{array}{l}\text { Large Cabbage } \\
\text { White }\end{array}$ & \\
\hline 51. Pieris napi montana & Green-veined White & \\
\hline 52. Pieris canidia indica & $\begin{array}{l}\text { Indian Cabbage } \\
\text { White }\end{array}$ & \\
\hline 53. Cepora nadina nadina & Lesser Gull & $\begin{array}{l}\text { Schedule II } \\
\text { Part II }\end{array}$ \\
\hline $\begin{array}{l}\text { 54. Prioneris thestylis } \\
\text { thestylis }\end{array}$ & Spotted Sawtooth & \\
\hline 55. Prioneris clemanthe & Redspot Sawtooth & \\
\hline 56. Delias belladonna lugens & Hill Jezebel & \\
\hline 57. Delias berinda & Dark Jezebel & \\
\hline 58. Delias acalis pyramus & Red-breast Jezebel & \\
\hline 59. Delias agostina agostina & Yellow Jezebel & \\
\hline \multicolumn{3}{|l|}{ Lycaenidae } \\
\hline 60. Curetis dentata dentata & Angled Sunbeam & \\
\hline 61. Taraka hamada mendesia & Forest Pierrot & \\
\hline 62. Caleta roxus roxana & Straight Pierrot & \\
\hline 63. Caleta elna noliteia & Elbowed Pierrot & \\
\hline $\begin{array}{l}\text { 64. Castalius rosimon } \\
\text { rosimon }\end{array}$ & Common Pierrot & $\begin{array}{l}\text { Schedule I } \\
\text { Part IV }\end{array}$ \\
\hline
\end{tabular}




\begin{tabular}{|c|c|c|}
\hline Scientific name & Common name & $\begin{array}{c}\text { Indian } \\
\text { WPA } 1972\end{array}$ \\
\hline 65. Tarucus indica & Pointed Pierrot & \\
\hline 66. Ancema ctesia ctesia & Bi-Spot Royal & \\
\hline 67. Remelana jangala ravata & Chocolate Royal & \\
\hline $\begin{array}{l}\text { 68. Arhopala centaurus } \\
\text { pirithous }\end{array}$ & Centaur Oakblue & \\
\hline 69. Flos adriana & $\begin{array}{l}\text { Variegated } \\
\text { Plushblue }\end{array}$ & \\
\hline 70. Flos asoka & Spangled Plushblue & \\
\hline 71. Surendra vivarna & $\begin{array}{l}\text { Common Acacia } \\
\text { Blue }\end{array}$ & \\
\hline 72. Zinaspa todara distorta & $\begin{array}{l}\text { Silver Streaked } \\
\text { Acacia Blue }\end{array}$ & $\begin{array}{l}\text { Schedule II } \\
\text { Part II }\end{array}$ \\
\hline $\begin{array}{l}\text { 73. Loxura atymnus } \\
\text { continentalis }\end{array}$ & Yamfly & \\
\hline $\begin{array}{l}\text { 74. Yasoda tripunctata } \\
\text { tripunctata }\end{array}$ & Branded Yamfly & $\begin{array}{l}\text { Schedule II } \\
\text { Part II }\end{array}$ \\
\hline 75. Cheritra freja freja & Common Imperial & \\
\hline 76. Ticherra acte & Blue Imperial & \\
\hline $\begin{array}{l}\text { 77. Hypolycaena erylus } \\
\text { himavantus }\end{array}$ & Common Tit & \\
\hline 78. Chliaria kina cachara & Blue Tit & $\begin{array}{c}\text { Schedule II } \\
\text { Part II }\end{array}$ \\
\hline 79. Zeltus amasa & Fluffy Tit & \\
\hline 80. Rapala nissa ratna & Common Flash & \\
\hline 81. Rapala manea schistacea & Slate Flash & \\
\hline 82. Catapoecilma elegans & Common Tinsel & \\
\hline $\begin{array}{l}\text { 83. Spindasis nipalicus } \\
\text { evansii }\end{array}$ & Silvergrey Silverline & $\begin{array}{c}\text { Schedule II } \\
\text { Part II }\end{array}$ \\
\hline 84. Spindasis rukmini & Khaki Silverline & $\begin{array}{l}\text { Schedule I } \\
\text { Part IV }\end{array}$ \\
\hline $\begin{array}{l}\text { 85. Spindasis lohita } \\
\text { himalayanus }\end{array}$ & $\begin{array}{l}\text { Long-banded } \\
\text { Silverline }\end{array}$ & $\begin{array}{c}\text { Schedule II } \\
\text { Part II }\end{array}$ \\
\hline 86. Heliophorus epicles & Purple Sapphire & \\
\hline $\begin{array}{l}\text { 87. Heliophorus brahma } \\
\text { major }\end{array}$ & Golden Sapphire & \\
\hline 88. Anthene emolus emolus & $\begin{array}{l}\text { Common Ciliate } \\
\text { Blue }\end{array}$ & \\
\hline $\begin{array}{l}\text { 89. Anthene lycaenina } \\
\text { lycaenina }\end{array}$ & Pointed Cilate Blue & \\
\hline 90. Leptotes plinius plinius & Zebra Blue & \\
\hline $\begin{array}{l}\text { 91. Nacaduba pactolus } \\
\text { continentalis }\end{array}$ & Large-4-Lineblue & $\begin{array}{c}\text { Schedule II } \\
\text { Part II }\end{array}$ \\
\hline 92. Nacaduba hermus nabo & Pale-4-Lineblue & $\begin{array}{c}\text { Schedule II } \\
\text { Part II }\end{array}$ \\
\hline 93. Nacaduba kurava euplea & $\begin{array}{l}\text { Transparent } \\
\text { 6-Lineblue }\end{array}$ & \\
\hline 94. Prosotas aluta coelestis & Banded Lineblue & \\
\hline 95. Prosotas nora nora & Common Lineblue & \\
\hline $\begin{array}{l}\text { 96. Lonolyce helicon } \\
\text { merguiana }\end{array}$ & Pointed Lineblue & \\
\hline 97. Petrelaea dana & Dingy Lineblue & \\
\hline 98. Jamides celeno celeno & Common Cerulean & \\
\hline 99. Jamides bochus bochus & Dark Cerulean & \\
\hline 100.Jamides elpis pseudelpis & Glistening Cerulean & $\begin{array}{l}\text { Schedule II } \\
\text { Part II }\end{array}$ \\
\hline
\end{tabular}

\begin{tabular}{|c|c|c|}
\hline Scientific name & Common name & $\begin{array}{c}\text { Indian } \\
\text { WPA } 1972\end{array}$ \\
\hline $\begin{array}{l}\text { 101. Jamides alecto } \\
\text { eurysaces }\end{array}$ & Metallic Cerulean & $\begin{array}{l}\text { Schedule II } \\
\text { Part II }\end{array}$ \\
\hline $\begin{array}{l}\text { 102. Catochrysops strabo } \\
\text { srrabo }\end{array}$ & Forget-me-not & \\
\hline 103. Lampides boeticus & Peablue & $\begin{array}{l}\text { Schedule II } \\
\text { Part II }\end{array}$ \\
\hline 104. Zizeeria karsandra & Dark Grass Blue & \\
\hline $\begin{array}{l}\text { 105. Pseudozizeeria maha } \\
\text { maha }\end{array}$ & Pale Grass Blue & \\
\hline 106. Freyeria putli & Eastern Grass Jewel & \\
\hline 107. Zizina otis otis & Lesser Grass Blue & \\
\hline 108. Megisba malaya & Malayan & $\begin{array}{l}\text { Schedule II } \\
\text { Part II }\end{array}$ \\
\hline 109. Acytolepis puspa gisca & $\begin{array}{l}\text { Common Hedge } \\
\text { Blue }\end{array}$ & $\begin{array}{l}\text { Schedule II } \\
\text { Part II }\end{array}$ \\
\hline 110. Udara cardia dilecta & Pale Hedge Blue & \\
\hline 111. Udara albocaerulea & Albocerulean & \\
\hline $\begin{array}{l}\text { 112. Celastrina lavendularis } \\
\text { limbata }\end{array}$ & Plain Hedge Blue & \\
\hline $\begin{array}{l}\text { 113. Celastrina argiolus } \\
\text { sikkima }\end{array}$ & Hill Hedge Blue & \\
\hline $\begin{array}{l}\text { 114. Celastrina huegelii } \\
\text { oreana }\end{array}$ & Large Hedge Blue & \\
\hline 115. Chilades laius laius & Lime Blue & \\
\hline 116. Abisara fylla & Dark Judy & \\
\hline 117. Zemeros flegyas indicus & Punchinello & \\
\hline 118. Dodonia adonira naga & Striped Punch & \\
\hline \multicolumn{3}{|l|}{ Nymphalidae } \\
\hline $\begin{array}{l}\text { 119. Libythea myrrha } \\
\text { sanguinalis }\end{array}$ & Club Beak & \\
\hline 120. Libythea lepita lepita & Common Beak & $\begin{array}{l}\text { Schedule II } \\
\text { Part II }\end{array}$ \\
\hline 121. Danaus genutia & Striped Tiger & \\
\hline $\begin{array}{l}\text { 122. Tirumala limniace } \\
\text { mutina }\end{array}$ & Blue Tiger & \\
\hline 123. Tirumala septentrionis & Dark Blue Tiger & \\
\hline $\begin{array}{l}\text { 124. Parantica aglea } \\
\text { melanoides }\end{array}$ & Glassy Tiger & \\
\hline 125. Parantica sita & Chestnut Tiger & \\
\hline $\begin{array}{l}\text { 126. Parantica melaneus } \\
\text { plateniston }\end{array}$ & Chocolate Tiger & \\
\hline $\begin{array}{l}\text { 127. Euploea mulciber } \\
\text { mulciber }\end{array}$ & Striped Blue Crow & $\begin{array}{l}\text { Schedule } \\
\text { IV }\end{array}$ \\
\hline 128. Euploea algea deione & $\begin{array}{l}\text { Long-branded Blue } \\
\text { Crow }\end{array}$ & \\
\hline $\begin{array}{l}\text { 129. Euploea midamus } \\
\text { splendens }\end{array}$ & Blue Spotted Crow & \\
\hline $\begin{array}{l}\text { 130. Euploea radamanthus } \\
\text { radamanthus }\end{array}$ & Magpie Crow & \\
\hline $\begin{array}{l}\text { 131. Charaxes athamas } \\
\text { athamas }\end{array}$ & Common Nawab & $\begin{array}{l}\text { Schedule II } \\
\text { Part II }\end{array}$ \\
\hline $\begin{array}{l}\text { 132. Charaxes eudamippus } \\
\text { eudamippus }\end{array}$ & Great Nawab & \\
\hline 133. Charaxes arja arja & Pallid Nawab & \\
\hline
\end{tabular}




\begin{tabular}{|c|c|c|}
\hline Scientific name & Common name & $\begin{array}{c}\text { Indian } \\
\text { WPA } 1972\end{array}$ \\
\hline $\begin{array}{l}\text { 134. Charaxes bernardus } \\
\text { hierax }\end{array}$ & Tawny Rajah & $\begin{array}{l}\text { Schedule II } \\
\text { Part II }\end{array}$ \\
\hline 135. Charaxes aristogiton & Scarce Tawny Rajah & $\begin{array}{l}\text { Schedule II } \\
\text { Part II }\end{array}$ \\
\hline 136. Charaxes kahruba & Variegated Rajah & $\begin{array}{l}\text { Schedule II } \\
\text { Part II }\end{array}$ \\
\hline $\begin{array}{l}\text { 137. Charaxes marmax } \\
\text { marmax }\end{array}$ & Yellow Rajah & $\begin{array}{l}\text { Schedule II } \\
\text { Part II }\end{array}$ \\
\hline $\begin{array}{l}\text { 138. Thaumantis diores } \\
\text { diores }\end{array}$ & Jungle Glory & \\
\hline 139. Faunis canens & Common Faun & \\
\hline 140. Discophora sondaica & Common Duffer & $\begin{array}{l}\text { Schedule I } \\
\text { Part IV }\end{array}$ \\
\hline 141. Melanitis leda & $\begin{array}{l}\text { Common Evening } \\
\text { Brown }\end{array}$ & \\
\hline 142. Lethe europa niladana & Bamboo Treebrown & $\begin{array}{l}\text { Schedule I } \\
\text { Part IV }\end{array}$ \\
\hline 143. Lethe mekara zuchara & $\begin{array}{l}\text { Common Red } \\
\text { Forester }\end{array}$ & \\
\hline 144. Lethe chandica flanona & Angled Red Forester & \\
\hline 145. Lethe sinorix & Tailed Red Forester & $\begin{array}{l}\text { Schedule II } \\
\text { Part II }\end{array}$ \\
\hline 146. Lethe gulnihal & Dull Forester & \\
\hline 147. Lethe sidonis sidonis & $\begin{array}{l}\text { Common } \\
\text { Woodbrown }\end{array}$ & \\
\hline 148. Lethe nicetella & Small Woodbrown & $\begin{array}{l}\text { Schedule II } \\
\text { Part II }\end{array}$ \\
\hline 149. Ethope himachala & Dusky Diadem & \\
\hline $\begin{array}{l}\text { 150. Penthema lisarda } \\
\text { lisarda }\end{array}$ & Yellow Kaiser & $\begin{array}{l}\text { Schedule II } \\
\text { Part II }\end{array}$ \\
\hline $\begin{array}{l}\text { 151. Elymnias hypermenestra } \\
\text { undularis }\end{array}$ & Common Palmfly & \\
\hline $\begin{array}{l}\text { 152. Elymnias malelas } \\
\text { malelas }\end{array}$ & Spotted Palmfly & \\
\hline $\begin{array}{l}\text { 153. Mycalesis anaxias } \\
\text { aemate }\end{array}$ & Whitebar Bushbrown & $\begin{array}{l}\text { Schedule II } \\
\text { Part II }\end{array}$ \\
\hline 154. Mycalesis adamsoni & Watson's Bushbrown & \\
\hline 155. Mycalesis malsarida & Plain Busbrown & $\begin{array}{l}\text { Schedule II } \\
\text { Part II }\end{array}$ \\
\hline $\begin{array}{l}\text { 156. Mycalesis perseus } \\
\text { blasius }\end{array}$ & Common Bushbrown & \\
\hline $\begin{array}{l}\text { 157. Mycalesis mineus } \\
\text { mineus }\end{array}$ & $\begin{array}{l}\text { Dark-brand } \\
\text { Bushbrown }\end{array}$ & $\begin{array}{l}\text { Schedule II } \\
\text { Part II? }\end{array}$ \\
\hline 158. Mycalesis visala visala & $\begin{array}{l}\text { Long-brand } \\
\text { Bushbrown }\end{array}$ & \\
\hline $\begin{array}{l}\text { 159. Mycalesis misenus } \\
\text { misenus }\end{array}$ & $\begin{array}{l}\text { Salmon-branded } \\
\text { Bushbrown }\end{array}$ & $\begin{array}{l}\text { Schedule II } \\
\text { Part II }\end{array}$ \\
\hline 160. Mycalesis nicotia & $\begin{array}{l}\text { Bright-eye } \\
\text { Bushbrown }\end{array}$ & \\
\hline $\begin{array}{l}\text { 161. Orsotrioena medus } \\
\text { medus }\end{array}$ & Nigger & \\
\hline 162. Ragadia crisilda crisilda & Striped Ringlet & $\begin{array}{l}\text { Schedule II } \\
\text { Part II }\end{array}$ \\
\hline 163. Zipoetis scylax & Dark Catseye & \\
\hline 164. Hemadara narasingha & Mottled Argus & \\
\hline 165. Ypthima nareda newara & Large Threering & \\
\hline 166. Ypthima baldus baldus & Common Fiverring & \\
\hline
\end{tabular}

\begin{tabular}{|c|c|c|}
\hline Scientific name & Common name & $\begin{array}{c}\text { Indian } \\
\text { WPA } 1972\end{array}$ \\
\hline 167. Acraea issoria issoria & Yellow Coster & \\
\hline 168. Acraea violae & Tawny Coster & \\
\hline 169. Cethosia biblis tisamena & Red Lacewing & \\
\hline 170. Cethosia cyane cyane & Leopard Lacewing & \\
\hline $\begin{array}{l}\text { 171. Argynnis hyperbius } \\
\text { hyperbius }\end{array}$ & Indian Fritillary & \\
\hline 172. Vindula erota erota & Cruiser & \\
\hline 173. Cirrochroa tyche mithila & Common Yeoman & \\
\hline 174. Cirrochroa aoris aoris & Large Yeoman & \\
\hline 175. Cupha erymanthis lotis & Rustic & \\
\hline 176. Vagrans egista sinha & Vagrant & \\
\hline $\begin{array}{l}\text { 177. Phalanta phalantha } \\
\text { phalantha }\end{array}$ & Common Leopard & \\
\hline 178. Sumalia daraxa daraxa & Green Commodore & \\
\hline 179. Auzakia danava danava & Commodore & \\
\hline 180. Parasarpa dudu dudu & White Commodore & \\
\hline 181. Limenitis zulema & $\begin{array}{l}\text { Scarce White } \\
\text { Commodore }\end{array}$ & $\begin{array}{l}\text { Schedule I } \\
\text { Part IV }\end{array}$ \\
\hline 182. Moduza procris procris & Commander & \\
\hline 183. Athyma asura asura & Studded Sergent & \\
\hline $\begin{array}{l}\text { 184. Athyma opalina } \\
\text { orientalis }\end{array}$ & Himalayan Sergent & \\
\hline 185. Athyma ranga ranga & Blackvein Sergent & $\begin{array}{l}\text { Schedule II } \\
\text { Part II }\end{array}$ \\
\hline $\begin{array}{l}\text { 186. Athyma selenophora } \\
\text { selenophora }\end{array}$ & Staff Sergent & \\
\hline 187. Athyma zeroca zeroca & Small Staff Sergent & \\
\hline 188. Athyma cama & $\begin{array}{l}\text { Orange Staff } \\
\text { Sergent }\end{array}$ & \\
\hline $\begin{array}{l}\text { 189. Pantoporia hordonia } \\
\text { hordonia }\end{array}$ & Common Lascar & \\
\hline 190. Pantoporia peraka & Perak Lascar & \\
\hline 191. Neptis radha radha & Great Yellow Sailer & $\begin{array}{l}\text { Schedule II } \\
\text { Part II }\end{array}$ \\
\hline 192. Neptis ananta ochracea & Yellow Sailer & $\begin{array}{c}\text { Schedule II } \\
\text { Part II }\end{array}$ \\
\hline 193. Neptis miah miah & Small Yellow Sailer & \\
\hline 194. Neptis manasa manasa & $\begin{array}{l}\text { Pale Hockeystick } \\
\text { Sailer }\end{array}$ & $\begin{array}{l}\text { Schedule I } \\
\text { Part IV }\end{array}$ \\
\hline 195. Neptis hylas astola & Common Sailer & \\
\hline 196. Neptis soma soma & Creamy Sailer & $\begin{array}{l}\text { Schedule II } \\
\text { Part II }\end{array}$ \\
\hline 197. Neptis clinia susruta & Sullied Sailer & \\
\hline 198. Neptis sankara amba & Broad-banded Sailer & $\begin{array}{l}\text { Schedule I } \\
\text { Part IV }\end{array}$ \\
\hline 199. Neptis pseudovikasi & Dingy Sailer & \\
\hline 200. Neptis cartica cartica & Plain Sailer & \\
\hline $\begin{array}{l}\text { 201. Phaedyma columella } \\
\text { ophiana }\end{array}$ & Short-banded Sailer & \\
\hline 202. Euthalia aconthea & Common Baron & \\
\hline 203. Euthalia lubentina & Gaudy Baron & $\begin{array}{l}\text { Schedule } \\
\text { IV }\end{array}$ \\
\hline
\end{tabular}




\begin{tabular}{|c|c|c|}
\hline Scientific name & Common name & $\begin{array}{c}\text { Indian } \\
\text { WPA } 1972\end{array}$ \\
\hline 204. Euthalia franciae & French Duke & $\begin{array}{l}\text { Schedule II } \\
\text { Part II }\end{array}$ \\
\hline 205. Lexias dirtea khasiana & Dark Archduke & \\
\hline $\begin{array}{l}\text { 206. Cyrestis thyodamas } \\
\text { thyodamas }\end{array}$ & Common Map & \\
\hline 207. Chersonesia risa & Common Maplet & \\
\hline 208. Pseudergolis wedah & Tabby & \\
\hline $\begin{array}{l}\text { 209. Dichorrhagia } \\
\text { nesimachus }\end{array}$ & Constable & \\
\hline 210. Stibochiona nicea & Popinjay & \\
\hline 211. Ariadne ariadne pallidior & Angled Castor & \\
\hline $\begin{array}{l}\text { 212.Ariadne merione } \\
\text { tapestrina }\end{array}$ & Common Castor & \\
\hline 213. Mimathyma chevana & Sergeant Emperor & $\begin{array}{l}\text { Schedule II } \\
\text { Part II }\end{array}$ \\
\hline 214. Mimathyma ambica & $\begin{array}{l}\text { Indian Purple } \\
\text { Emperor }\end{array}$ & \\
\hline 215. Euripus nyctelius & Courtesan & \\
\hline 216. Hestinalis nama & Circe & \\
\hline 217. Sephisa chandra & Eastern Courtier & $\begin{array}{l}\text { Schedule I } \\
\text { Part IV }\end{array}$ \\
\hline $\begin{array}{l}\text { 218. Symbrenthia lilaea } \\
\text { khasiana }\end{array}$ & Common Jester & \\
\hline $\begin{array}{l}\text { 219. Symbrenthia hypselis } \\
\text { cotanda }\end{array}$ & Spotted Jester & \\
\hline 220. Vanessa indica indica & Indian Red Admiral & \\
\hline 221. Vanessa cardui & Painted Lady & \\
\hline 222. Kaniska canace canace & Blue Admiral & \\
\hline 223. Rohana parisatis & Black Prince & \\
\hline 224. Rohana parvata & Brown Prince & $\begin{array}{l}\text { Schedule II } \\
\text { Part II }\end{array}$ \\
\hline 225. Junonia iphita iphita & Chocolate Pansy & \\
\hline 226. Junonia atlites & Grey Pansy & \\
\hline 227. Junonia almana almana & Peacock Pansy & \\
\hline $\begin{array}{l}\text { 228. Junonia lemonias } \\
\text { lemonias }\end{array}$ & Lemon Pansy & \\
\hline 229. Hypolimnas bolina & Great Eggfly & \\
\hline 230. Kallima inachus inachus & Orange Oakleaf & \\
\hline $\begin{array}{l}\text { 231. Doleschallia bisaltide } \\
\text { indica }\end{array}$ & Autumn Leaf & $\begin{array}{l}\text { Schedule I } \\
\text { Part IV }\end{array}$ \\
\hline 232. Neurosigma doubledayi & Panther & \\
\hline \multicolumn{3}{|l|}{ Hesperiidae } \\
\hline $\begin{array}{l}\text { 233. Burara oedipodea } \\
\text { aegina }\end{array}$ & $\begin{array}{l}\text { Branded Orange } \\
\text { Awlet }\end{array}$ & \\
\hline 234. Burara jaina vasundhara & Orange Awlet & \\
\hline 235. Burara amara & Small Green Awlet & \\
\hline 236. Burara vasutana & Green Awlet & \\
\hline 237. Burara gomata gomata & Pale Green Awlet & \\
\hline 238. Hasora anura danda & Slate Awl & \\
\hline
\end{tabular}

\begin{tabular}{|c|c|c|}
\hline Scientific name & Common name & $\begin{array}{c}\text { Indian } \\
\text { WPA } 1972\end{array}$ \\
\hline 239. Hasora badra badra & Common Awl & \\
\hline 240. Hasora vita indica & Plain Banded Awl & \\
\hline 241. Hasora chromus & $\begin{array}{l}\text { Common Banded } \\
\text { Awl }\end{array}$ & \\
\hline $\begin{array}{l}\text { 242. Hasora taminatus } \\
\text { bhavara }\end{array}$ & White-banded Awl & \\
\hline 243. Badamia exclamationis & Brown Awl & \\
\hline 244. Bibasis sena sena & Orange-tail Awl & $\begin{array}{l}\text { Schedule II } \\
\text { Part II }\end{array}$ \\
\hline 245. Choaspes benjaminii & Indian Awlking & \\
\hline $\begin{array}{l}\text { 246. Celaenorrhinus } \\
\text { leucocera chinensis }\end{array}$ & $\begin{array}{l}\text { Common Spotted } \\
\text { Flat }\end{array}$ & \\
\hline $\begin{array}{l}\text { 247. Celaenorrhinus } \\
\text { aurivittata aurivittata }\end{array}$ & $\begin{array}{l}\text { Dark Yellow-banded } \\
\text { Flat }\end{array}$ & \\
\hline 248. Seseria dohertyi & $\begin{array}{l}\text { Himalayan White } \\
\text { Flat }\end{array}$ & \\
\hline 249. Pseudocoladenia dan & Fulvous Pied Flat & \\
\hline 250. Darpa hanria & Hairy Angle & \\
\hline 251. Gerosis sinica indica & $\begin{array}{l}\text { White Yellow-breast } \\
\text { Flat }\end{array}$ & \\
\hline $\begin{array}{l}\text { 252. Tagiades litigiosa } \\
\text { litigiosa }\end{array}$ & Water Snow Flat & \\
\hline $\begin{array}{l}\text { 253. Mooreana trichoneura } \\
\text { pralaya }\end{array}$ & Yellow Flat & \\
\hline 254. Odontoptilum angulata & Chestnut Angle & \\
\hline $\begin{array}{l}\text { 255. Ctenoptilum vasava } \\
\text { vasava }\end{array}$ & Tawny Angle & \\
\hline 256. Suastus minuta aditia & $\begin{array}{l}\text { Small Indian Palm } \\
\text { Bob }\end{array}$ & \\
\hline 257. Oriens gola gola & Common Dartlet & \\
\hline 258. Potanthus zatilla & Common Dart & \\
\hline $\begin{array}{l}\text { 259. Potanthus trachala } \\
\text { tytleri }\end{array}$ & Broad Bident Dart & \\
\hline 260. Potanthus tropica & Tropic Dart & \\
\hline 261. Potanthus mara & Sikkim Dart & \\
\hline 262. Telicota augias & Pale Palm Dart & \\
\hline 263. Telicota ancilla horisha & Dark Palm Dart & \\
\hline $\begin{array}{l}\text { 264. Pithauria } \\
\text { stramineipennis } \\
\text { stramineipennis }\end{array}$ & Light Straw Ace & \\
\hline 265. Pithauria murdava & Dark Straw Ace & \\
\hline 266. Thoressa hyrie & Chequered Ace & \\
\hline \multicolumn{3}{|l|}{ 267. Thoressa sp. } \\
\hline 268. Thoressa cerata & $\begin{array}{l}\text { Northern Spotted } \\
\text { Ace }\end{array}$ & \\
\hline 269. Halpe zema zema & Banded Ace & \\
\hline 270. Halpe porus & Moore's Ace & \\
\hline 271. Halpe kumara & Plain Ace & \\
\hline 272. Halpe kusala & Hill Ace & \\
\hline 273. Sebastonyma dolopia & Tufted Ace & \\
\hline 274. Caltoris pagana & Figure of 8 Swift & \\
\hline 275. Caltoris cahira cara & Colon Swift & \\
\hline
\end{tabular}




\begin{tabular}{|l|l|l|}
\hline Scientific name & Common name & $\begin{array}{c}\text { Indian } \\
\text { WPA 1972 }\end{array}$ \\
\hline 276. Baoris farri & Paintbrush Swift & $\begin{array}{c}\text { Schedule } \\
\text { IV }\end{array}$ \\
\hline 277. Polytremis lubricans & Contiguous Swift & \\
\hline 278. Polytremis eltola & Yellow-Spot Swift & \\
\hline 279. Parnara sp. & & \\
\hline 280. Pseudoborbo bevani & Bevan's Swift & \\
\hline 281. Hyarotis adrastus praba & Tree Flitter & \\
\hline 282. Zographetus satwa & $\begin{array}{l}\text { Purple and Gold } \\
\text { Flitter }\end{array}$ & \\
\hline 283. Matapa sasivarna & $\begin{array}{l}\text { Black-veined } \\
\text { Redeye }\end{array}$ & \\
\hline $\begin{array}{l}\text { 284. Baracus vittatus } \\
\text { septentrionum }\end{array}$ & Hedge Hopper & \\
\hline $\begin{array}{l}\text { 285. Scobura cephaloides } \\
\text { cephaloides }\end{array}$ & Large Forest Bob & \\
\hline
\end{tabular}

\begin{tabular}{|l|l|l|}
\hline Scientific name & Common name & $\begin{array}{c}\text { Indian } \\
\text { WPA 1972 }\end{array}$ \\
\hline $\begin{array}{l}\text { 286. Koruthaialos butleri } \\
\text { butleri }\end{array}$ & Dark Velvet Bob & \\
\hline 287. Ancistroides nigrita & Chocolate Demon & \\
\hline $\begin{array}{l}\text { 288. Notocrypta paralysos } \\
\text { alysia }\end{array}$ & $\begin{array}{l}\text { Common Banded } \\
\text { Demon }\end{array}$ & \\
\hline $\begin{array}{l}\text { 289. Notocrypta feisthameli } \\
\text { alysos }\end{array}$ & Spotted Demon & \\
\hline 290. Udaspes folus & Grass Demon & \\
\hline 291. Asticopterus jama kada & Forest Hopper & \\
\hline $\begin{array}{l}\text { 292. Aeromachus stigmata } \\
\text { stigmata }\end{array}$ & $\begin{array}{l}\text { Veined Scrub } \\
\text { Hopper }\end{array}$ & \\
\hline 293. Aeromachus jhora creta & Grey Scrub Hopper & \\
\hline 294. Baracus sp. & \multicolumn{2}{|l}{} \\
\hline
\end{tabular}

be numerous additions to the following list when surveys are conducted at other seasons. Also, much of the study was concentrated in and around Dibang River bed, the lowest altitude of Mishmi Hills. Very little survey was done between Tiwarigaon $(1,300 \mathrm{~m})$ and Mayodia Pass $(2,666 \mathrm{~m})$, proper survey in this area will yield more species.

\section{REFERENCES}

Champion H.G. \& S.K. Seth (1968). A Revised Survey of the Forest Types of India. The Manager of Publucations. Government of India, New Delhi, 404pp.

Evans, W.H. (1932). The Identification of Indian Butterflies-

2nd Edition. Bombay Natural History Society, Mumbai, India, 464pp+32pl.

Haribal, M. (1992) The Butterflies of Sikkim Himalaya. Sikkim Nature Conservation Foundation, Sikkim, India. 217pp.

Kehimkar, I. (2008). The Book of Indian Butterflies. Bombay Natural History Society and Oxford University Press, Mumbai, India, 497pp.

Smith, C. (1994). Butterflies of Nepal. Tecpress Services L.P., Bangkok, Thailand, 368pp.

Talbot, G. (1947). The Fauna of British India, including Ceylon and Burma: Butterflies, Vol. II. Taylor and Francis, London. 506pp.

Watson, E.Y. (1897). Hesperiidae Indicae : being a reprint of descriptions of the Hesperiidae of India, Burma, and Ceylon. Madras Staff Corps, Vest and Company Mount Road, Madras, 161pp.

(C) All photos by Monsoon J. Gogoi, except for Image 33, which is by Rajib R Tariang. 


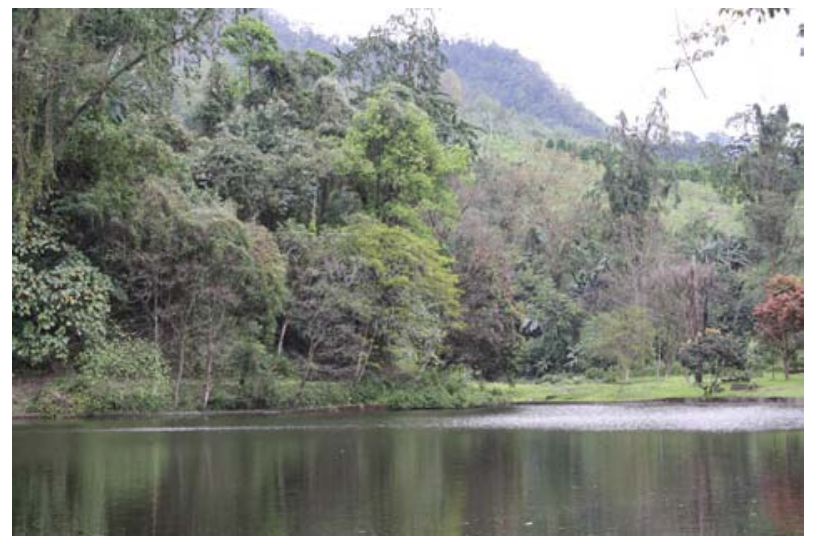

Image 2. Sally Lake near Roing Town

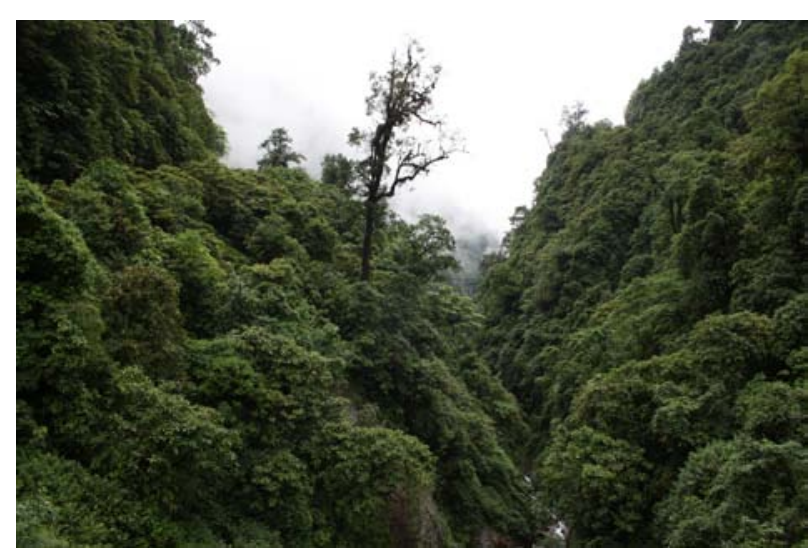

Image 4a. Forest in Tiwarigaon

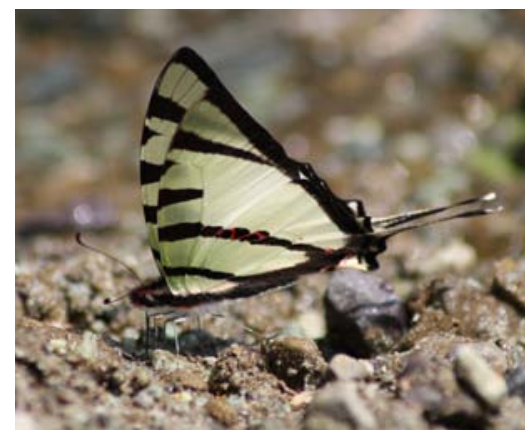

Image 5. Graphium agetes agetes

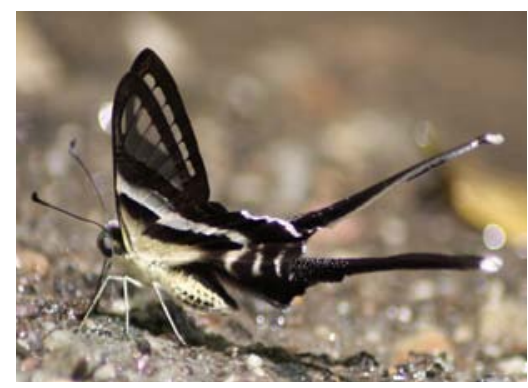

Image 8. Lamproptera curius curius pompilius

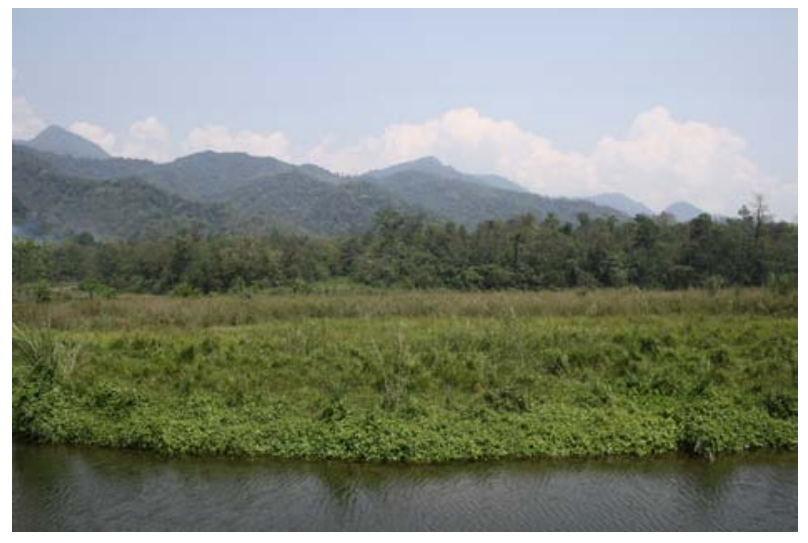

Image 3. Mishmi Hills from Dibang River Bed

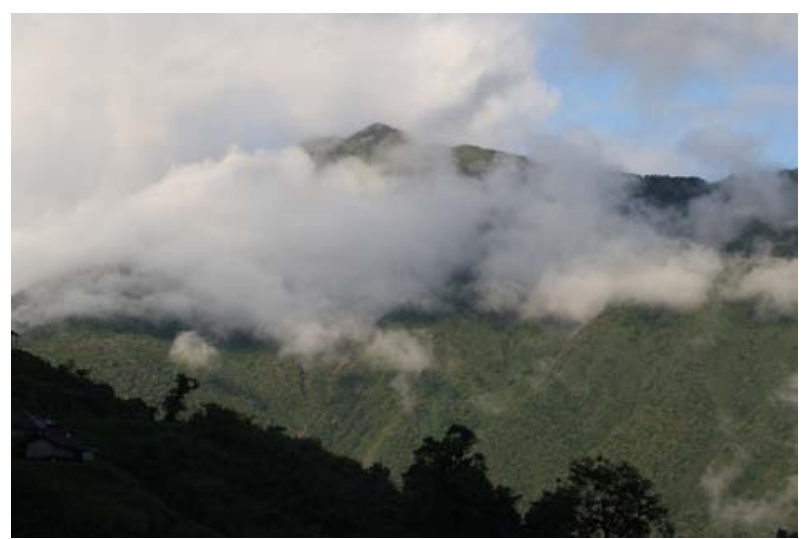

Image 4b. View of Mayodia

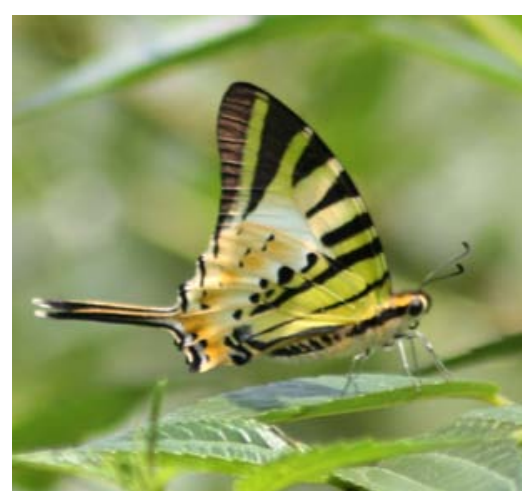

Image 6. Graphium antiphates

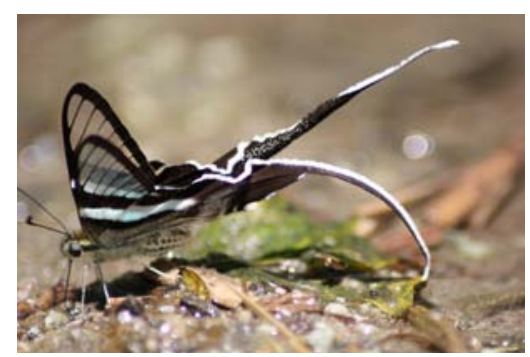

Image 7. Lamproptera meges indistincta

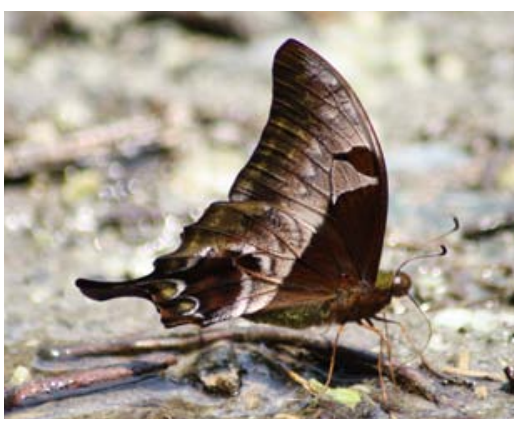

Image 9. Lamproptera curius curius

Journal of Threatened Taxa | www.threatenedtaxa.org | October 2012 | 4(12): 3137-3160 


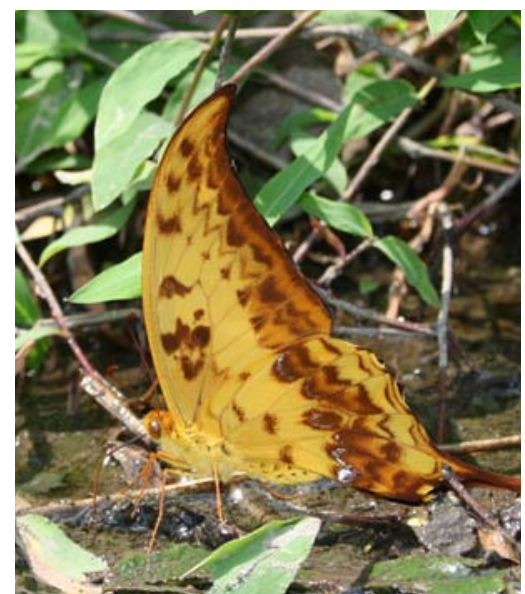

Image 10. Meandrusa payeni evan

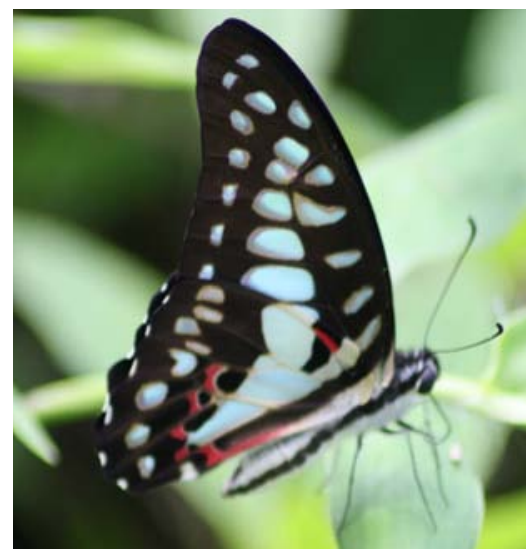

Image 13. Graphium eurypylus acheron

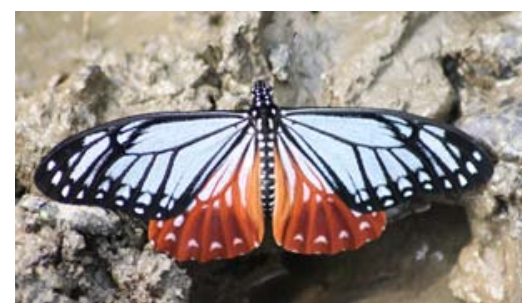

Image 16. Papilio agestor agestor

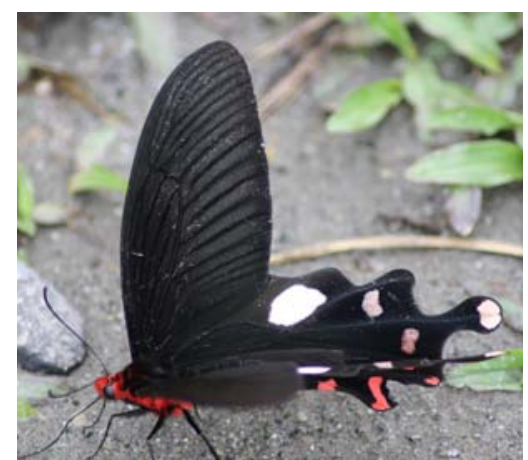

Image 19. Byasa dasarada dasarada

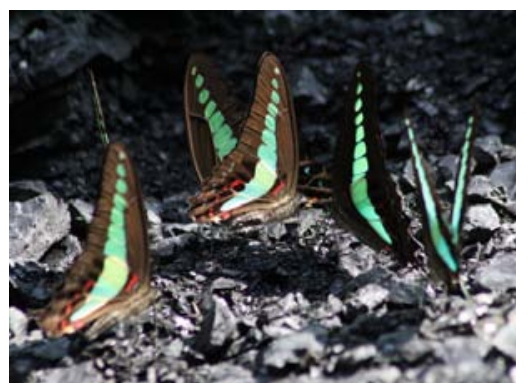

Image 11. Graphium sarpedon sarpedon

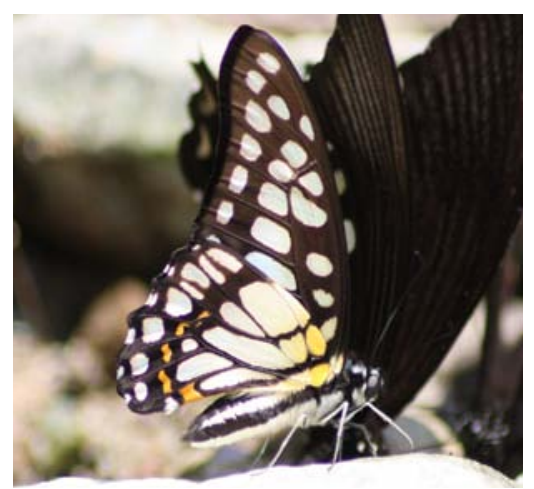

Image 14. Graphium chironides chironides

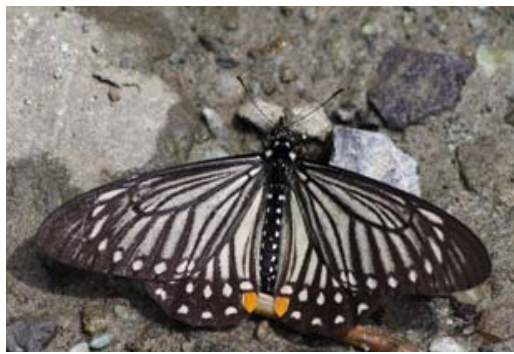

Image 17. Papilio epycides epycides

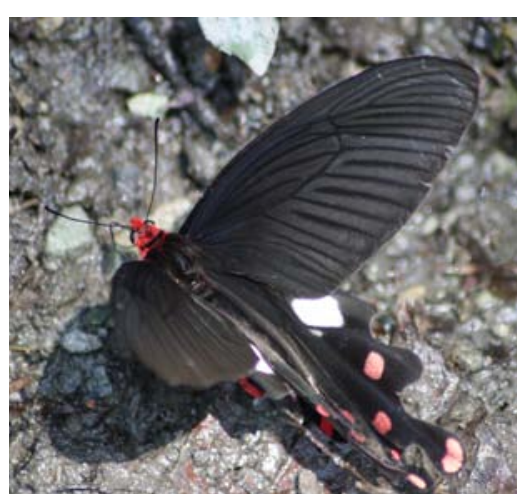

Image 20. Byasa philoxenus polyeuctes

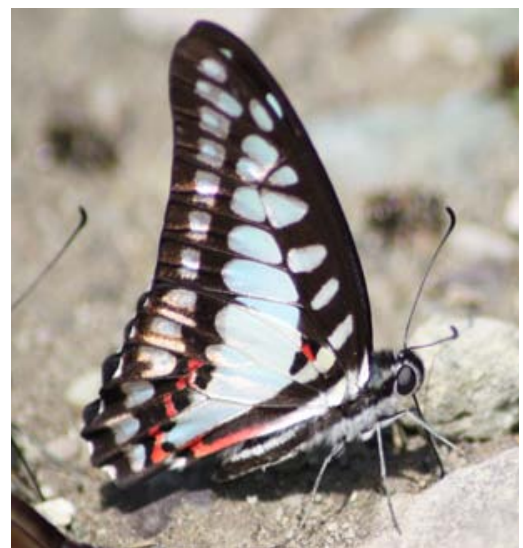

Image 12. Graphium evemon albociliatis

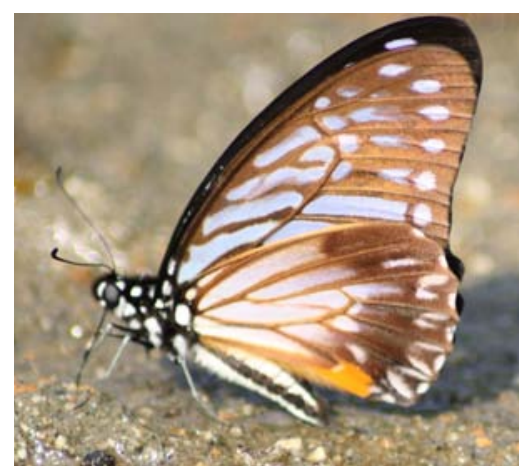

Image 15. Graphium xenocles xenocles

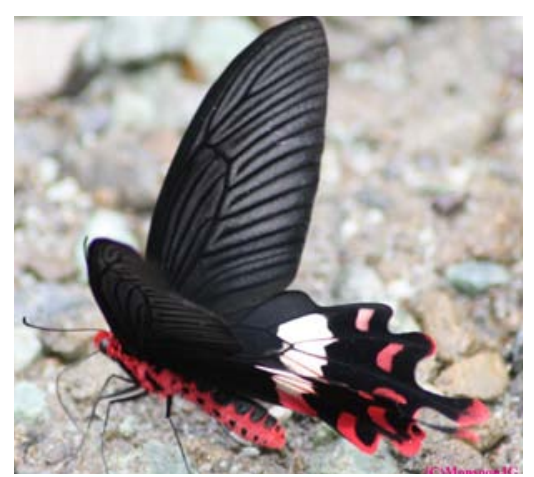

Image 18. Byasa polla 


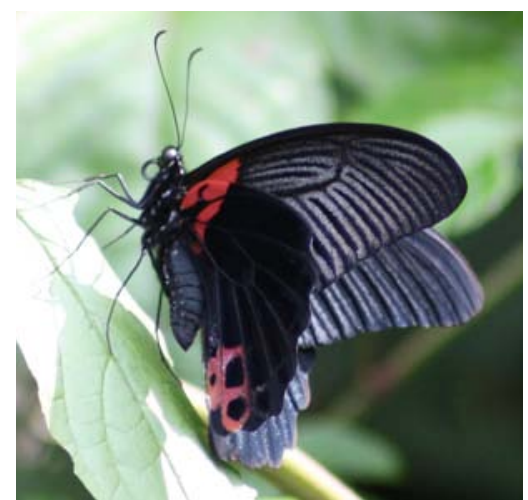

Image 22. Papilio memnon agenor

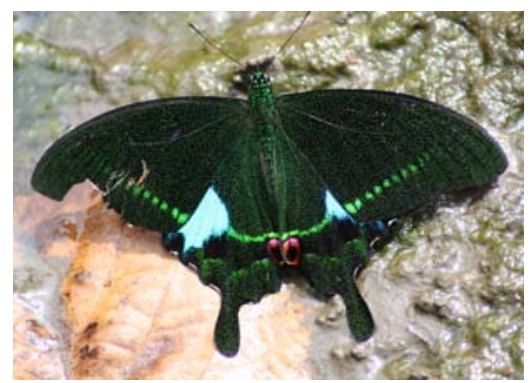

Image 24. Papilio paris paris

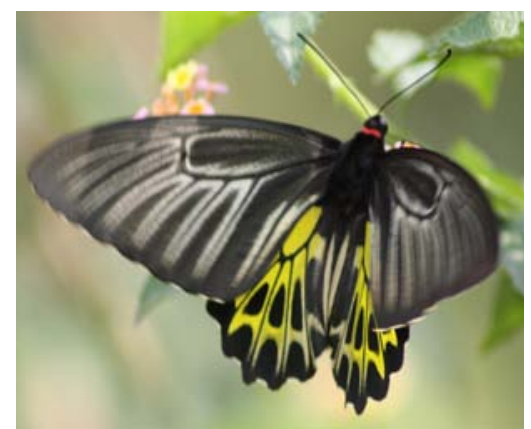

Image 27. Troides aeacus (female)

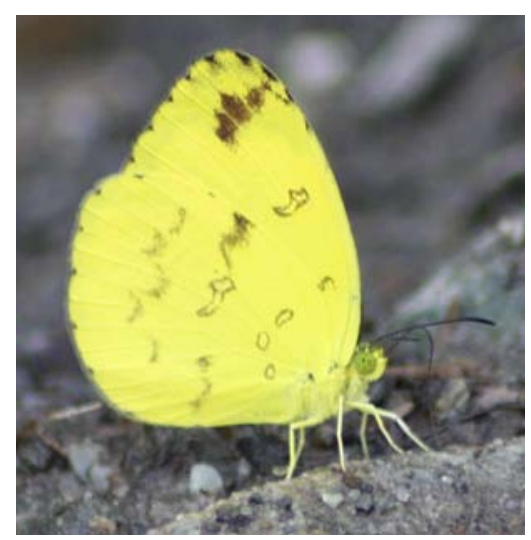

Image 30. Eurema andersoni andersoni

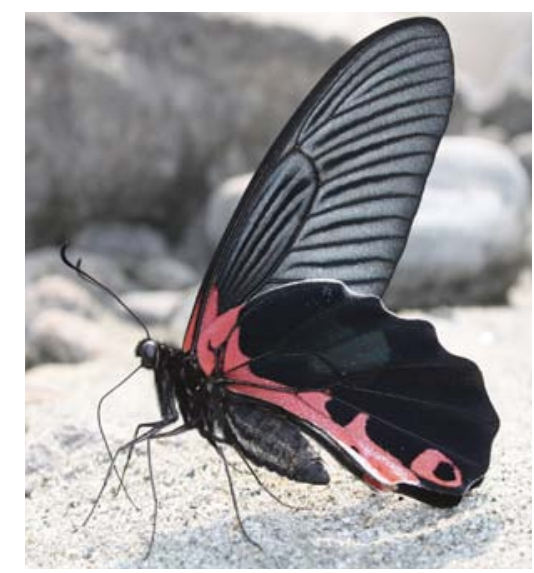

Image 23. Papilio alcmenor alcmenor

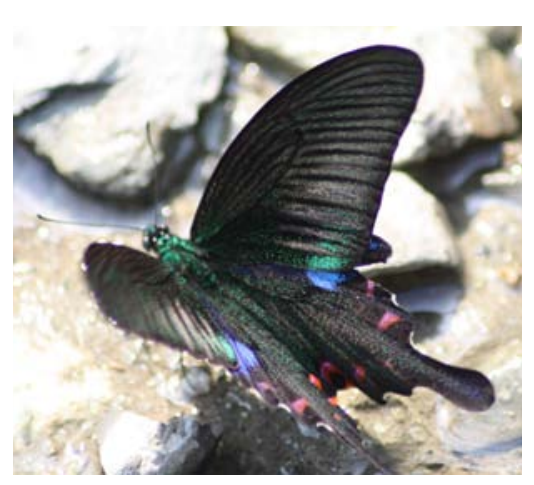

Image 25. Papilio polyctor ganesa

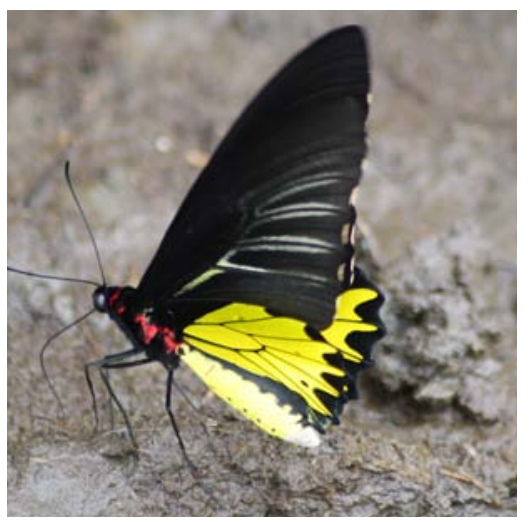

Image 28. Troides helena cerberus(male)

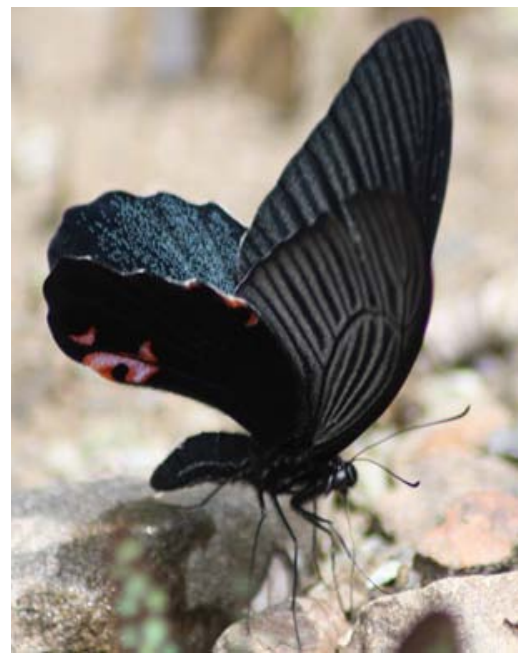

Image 21. Papilio protenor euprotenor

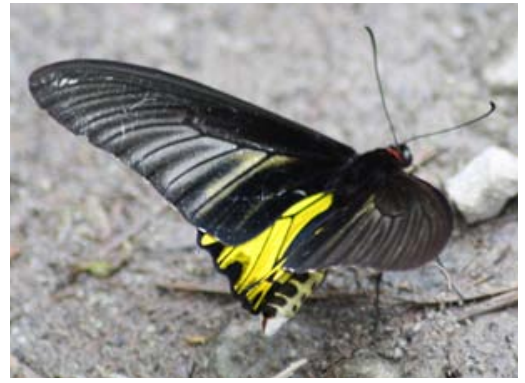

Image 26. Troides aeacus (male)

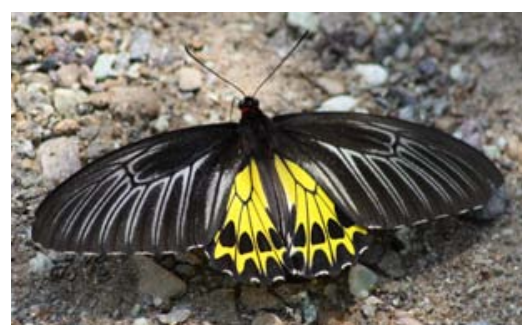

Image 29. Troides helena cerberus (female)

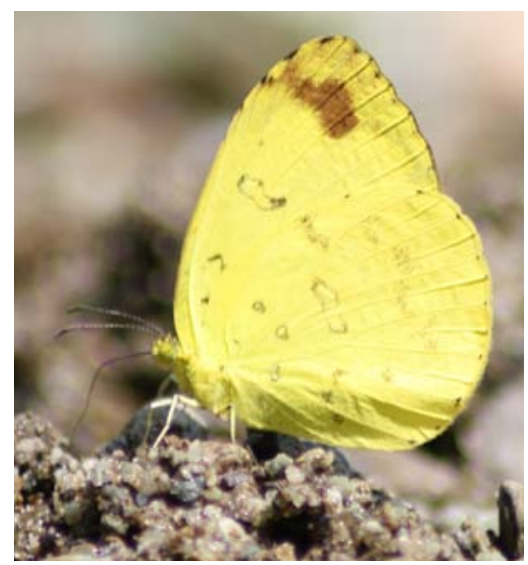

Image 31. Eurema hecabe simulata 


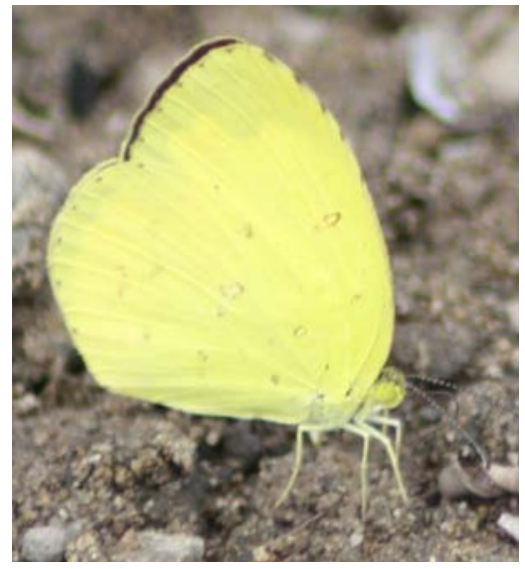

Image 32. Eurema brigitta rubella

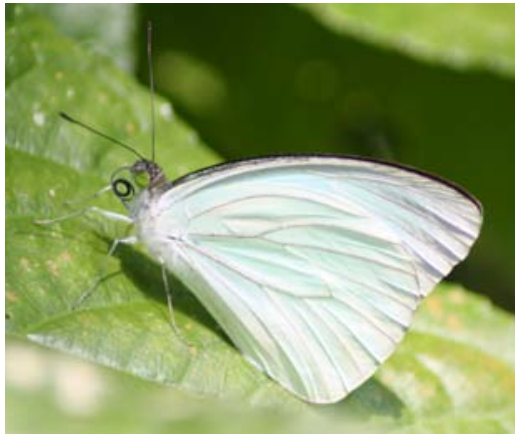

Image 35. Pareronia avatar avatar

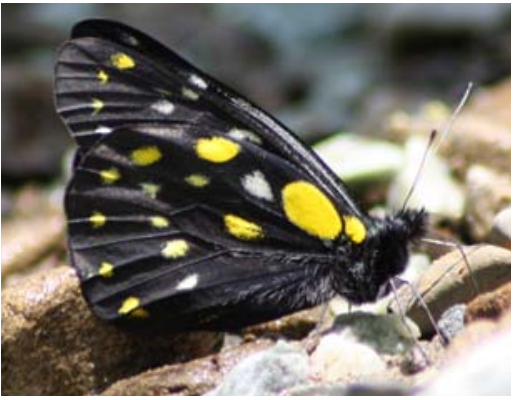

Image 38. Delias berinda berinda

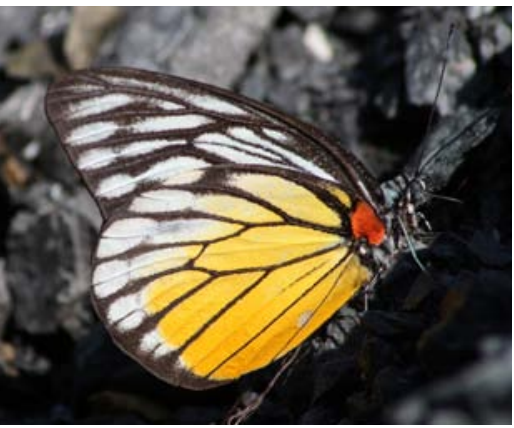

Image 41. Prioneris clemanthe

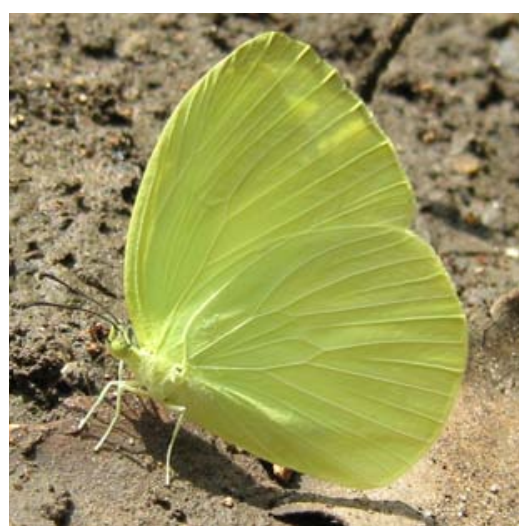

Image 33. Gandaca harina assamica

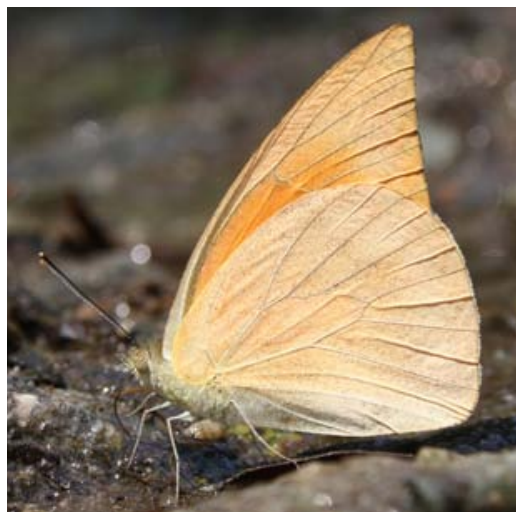

Image 36. Appias nero galba

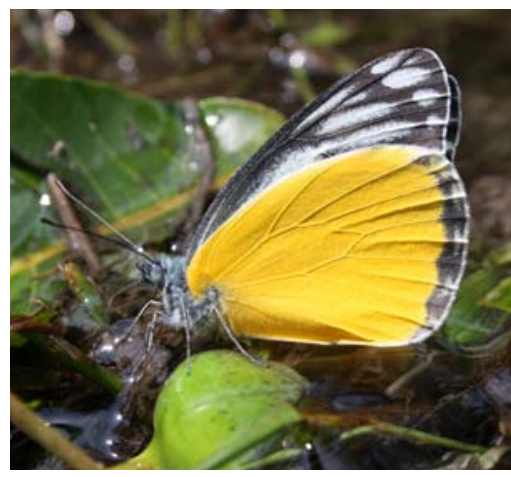

Image 39. Delias agostina

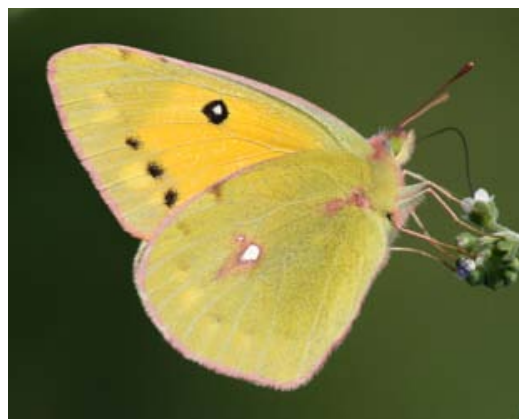

Image 42. Colias fieldii fieldii

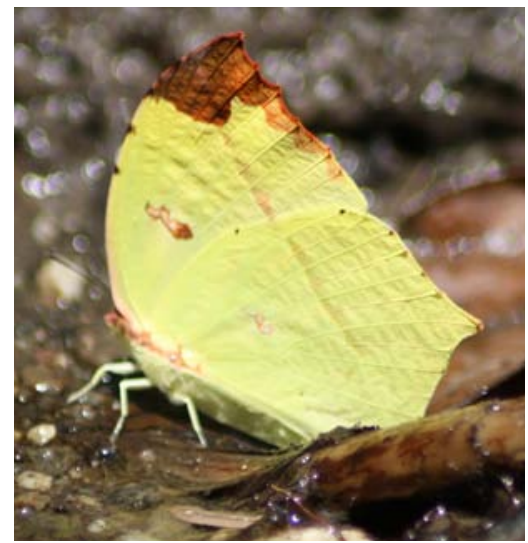

Image 34. Dercas verhuelli doubledayi

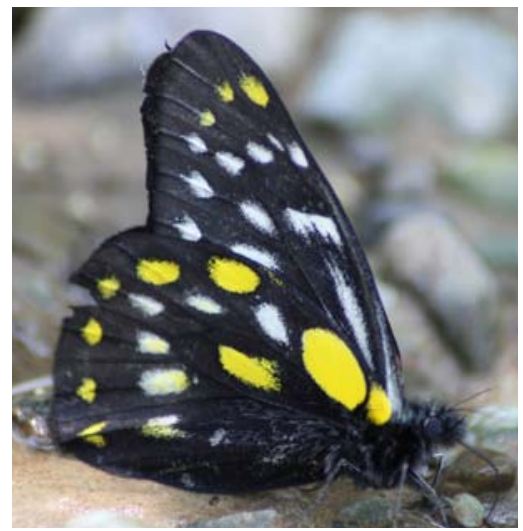

Image 37. Delias belladonna lugens

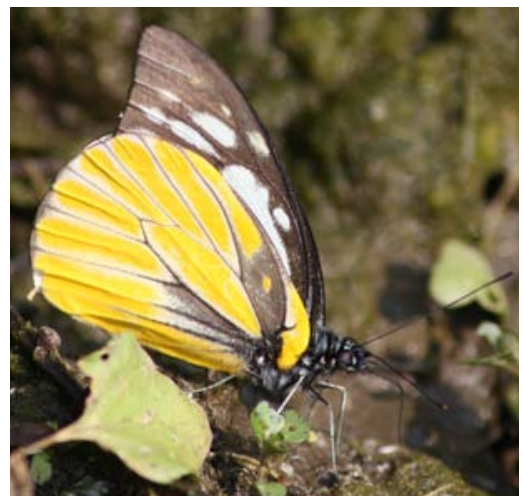

Image 40. Prioneris thestylis thestylis

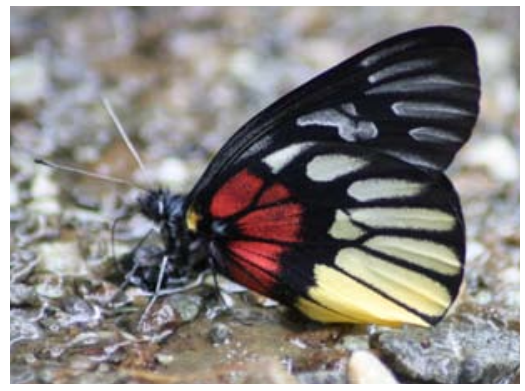

Image 43. Delias thysbe pyramus 


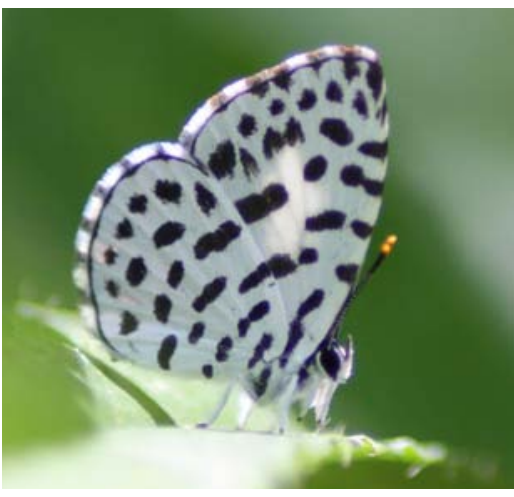

Image 44. Taraka hamada mendesia

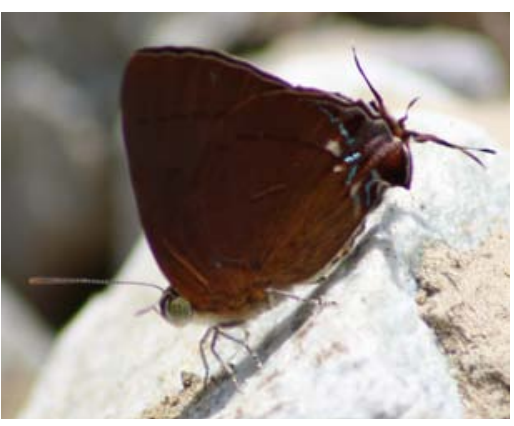

Image 47. Remelana jangala ravata

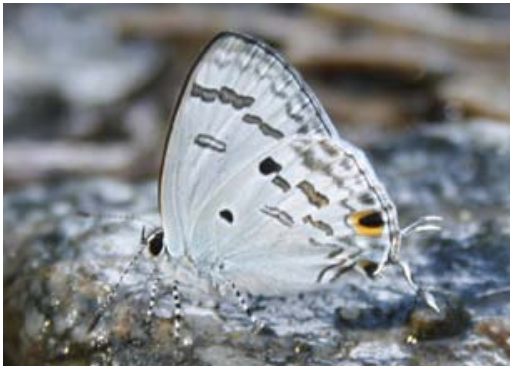

Image 50. Chilaria kina cachara

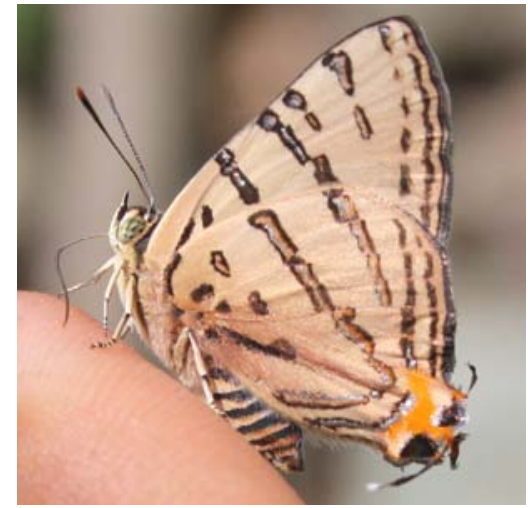

Image 53. Spindasis rukmini

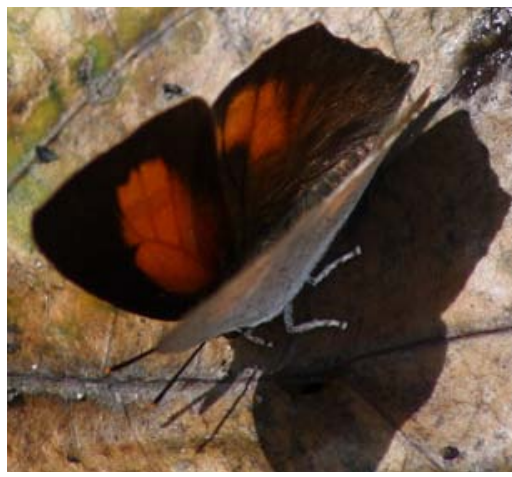

Image 45. Curetis dentata dentata

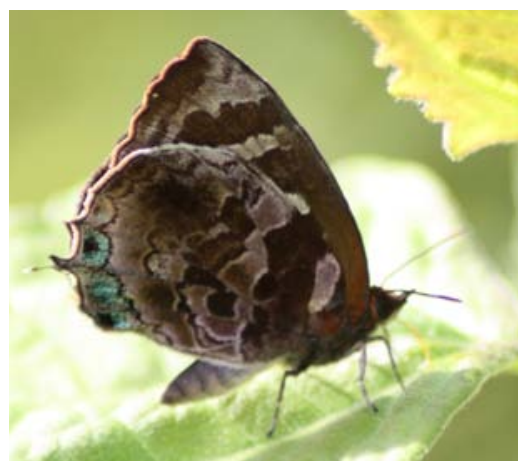

Image 48. Flos asoka

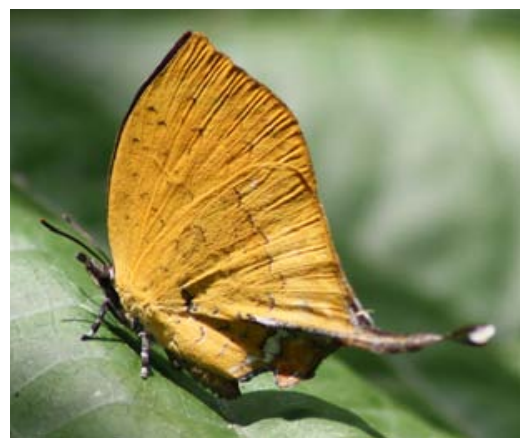

Image 51. Yasoda tripunctata tripunctata

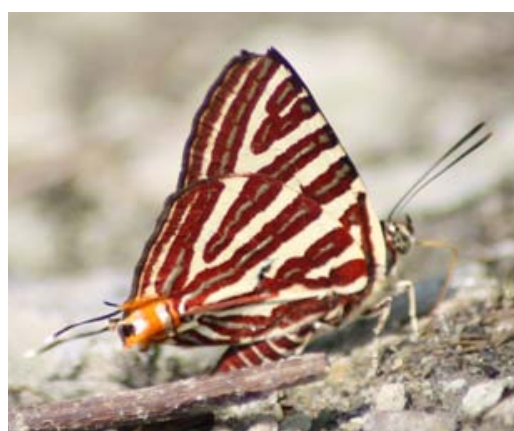

Image 54. Spindasis lohita himalayanus

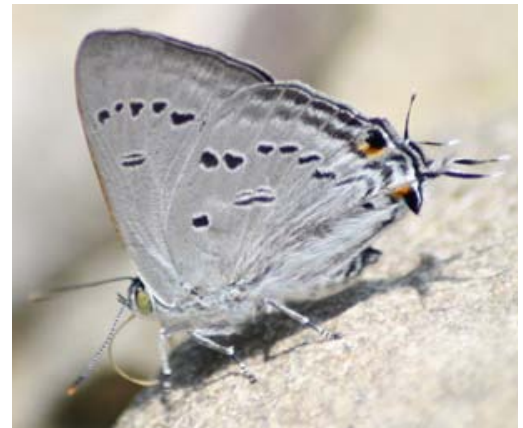

Image 46. Ancema ctesia ctesia

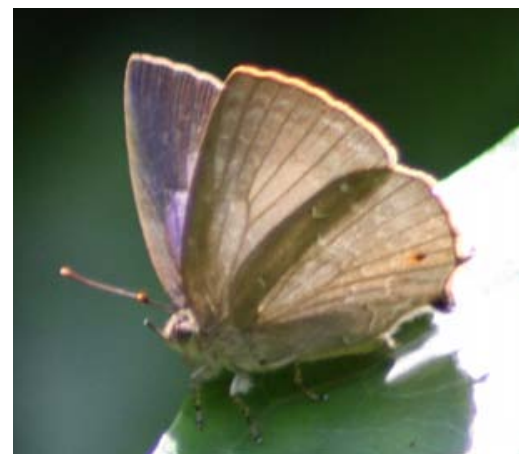

Image 49. Zanaspa todara distorta

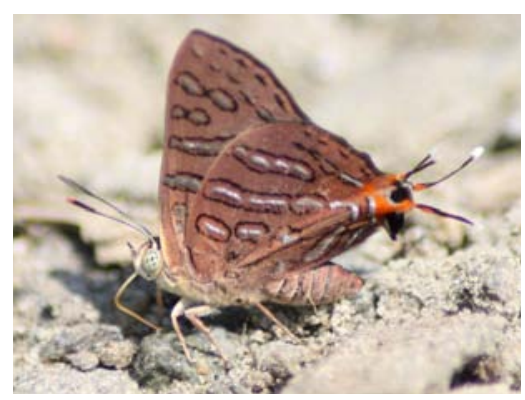

Image 52. Spindasis nipalicus evansii

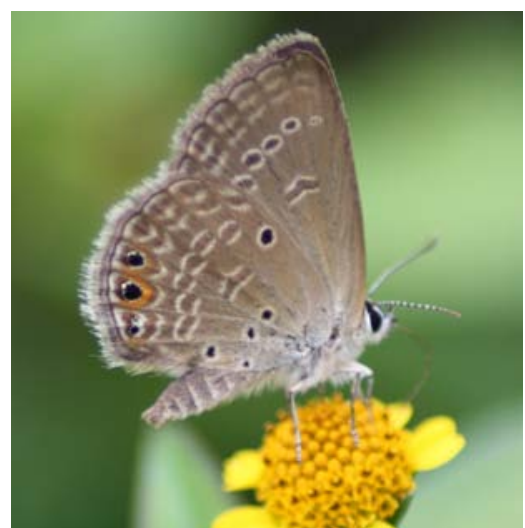

Image 55. Freyeria putli 


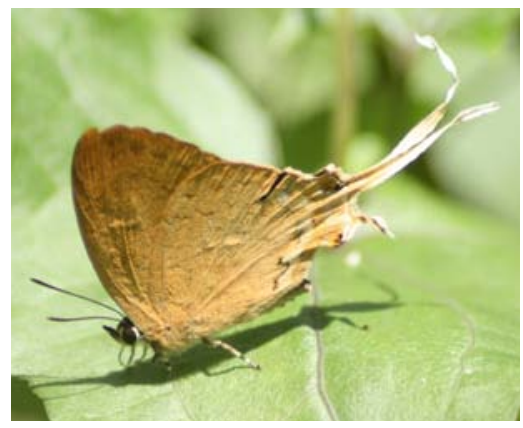

Image 56. Ticherra acte

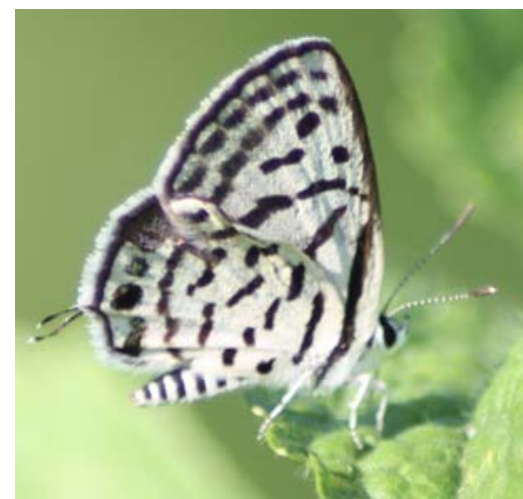

Image 59. Tarucus indica

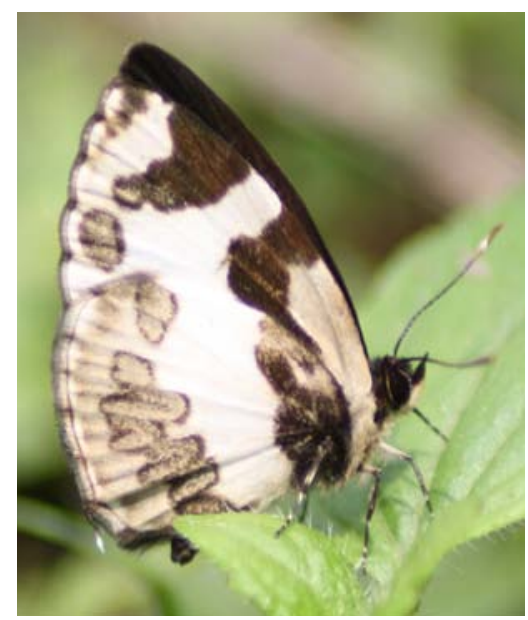

Image 62. Caleta elna noliteia

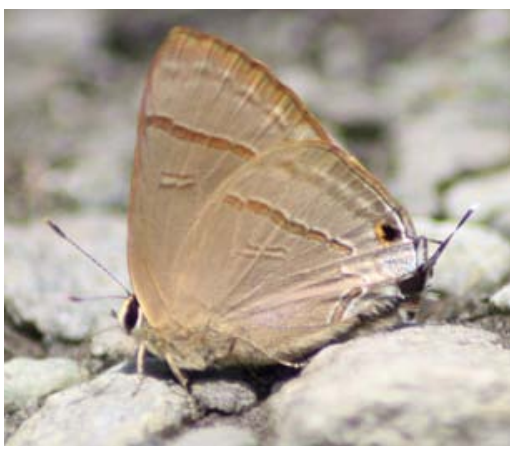

Image 57. Rapala nissa ratna

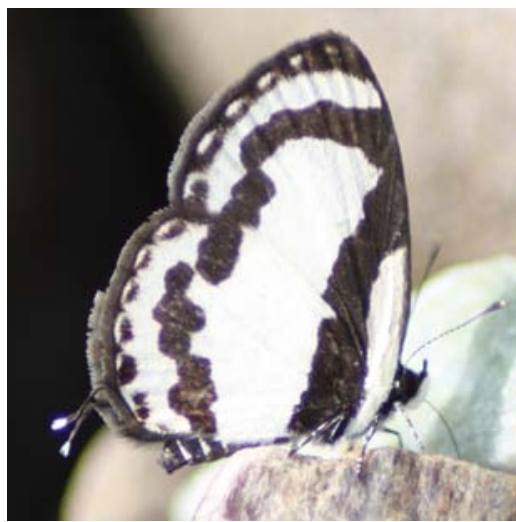

Image 60. Caleta roxus roxona

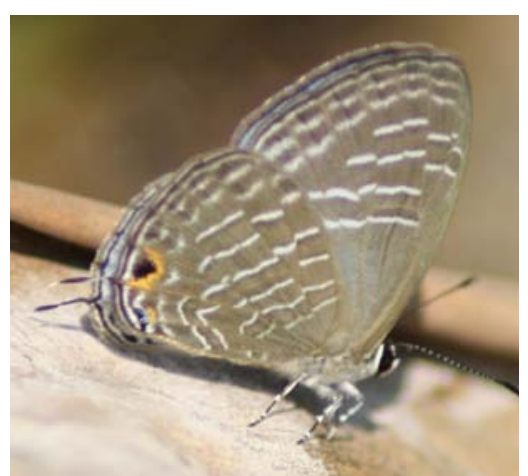

Image 63. Jamides elepis pseudelpis

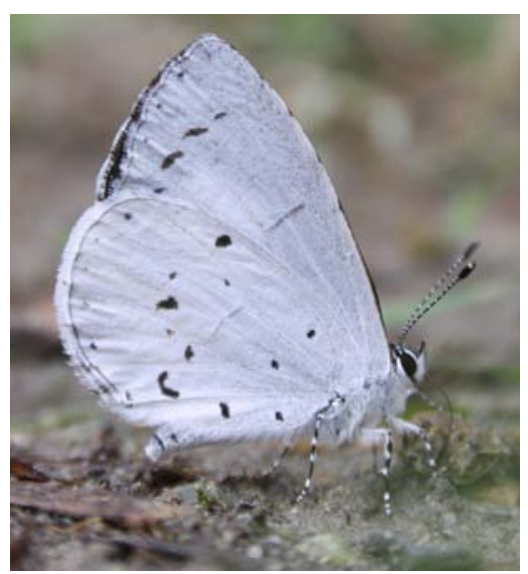

Image 65. Udara albocaerulea

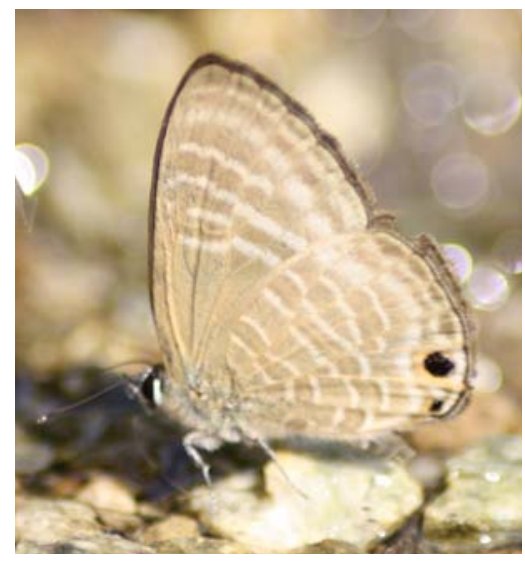

Image 58. Nacaduba hermus nabo

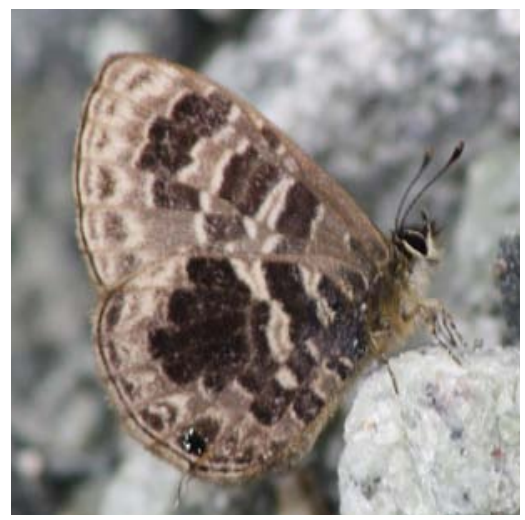

Image 61. Prosotas aluta coelestis

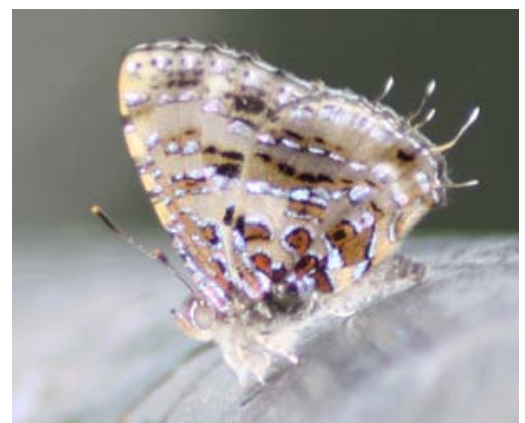

Image 64. Catapoecilma elegans 


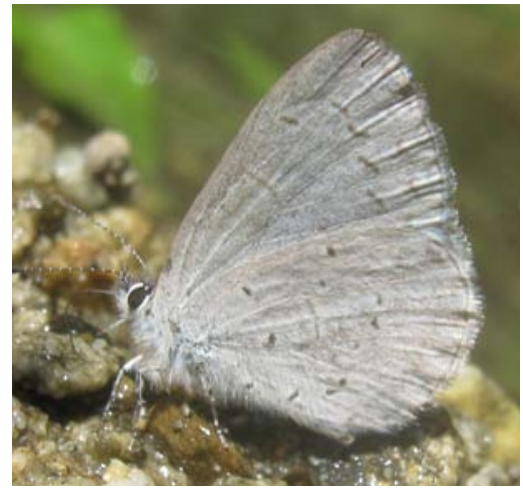

Image 66. Udara cardia dilecta

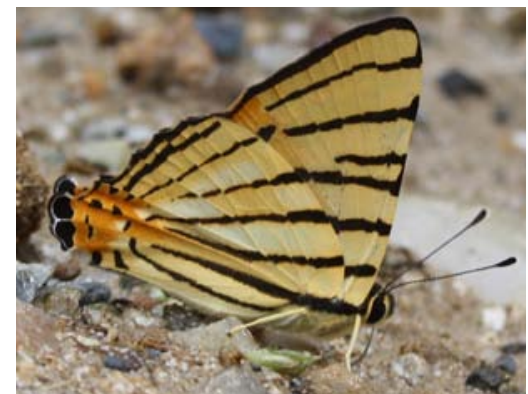

Image 69. Dodonia adonira naga

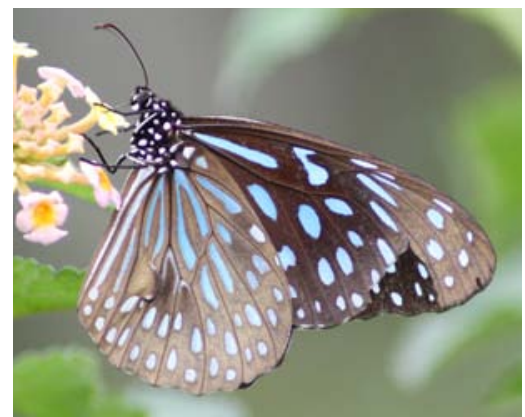

Image 72. Danais melissa septentrionis

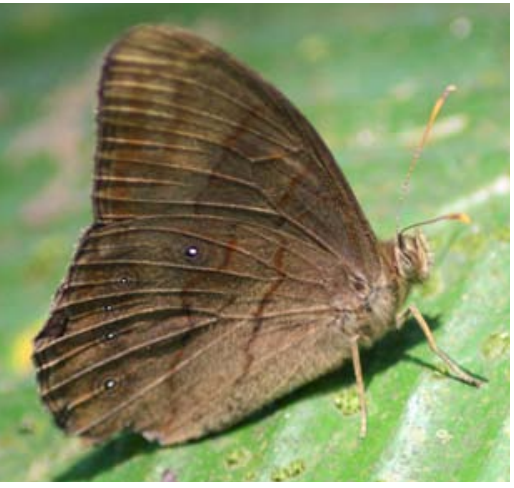

Image 75. Lethe gulnihal

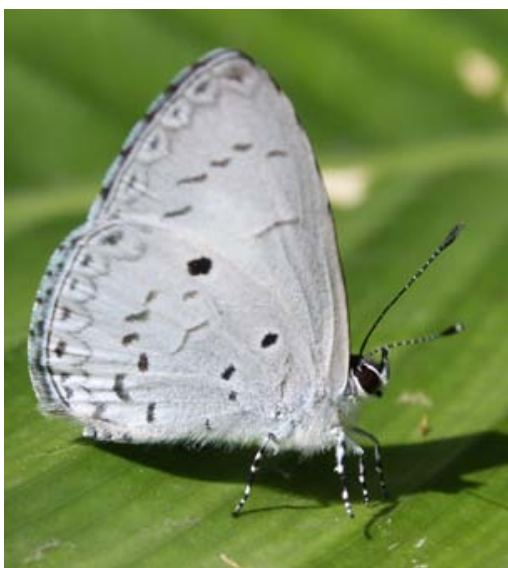

Image 67. Celastrina argiolus sikkima

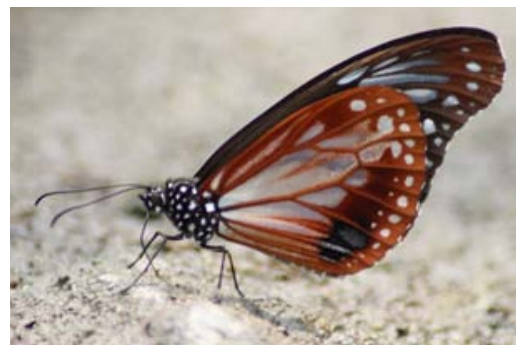

Image 70. Parantica tytia sita

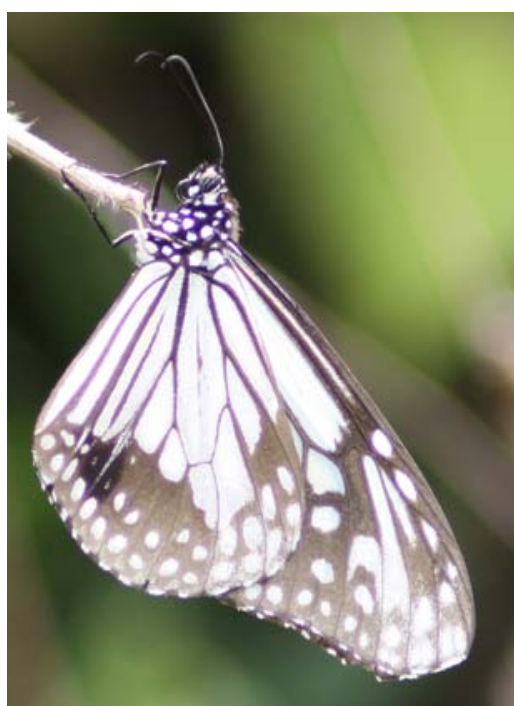

Image 73. Parantica aglea melanoides

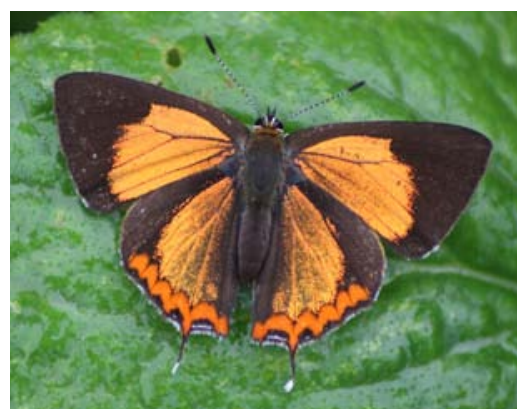

Image 68. Heliophorus brahma major

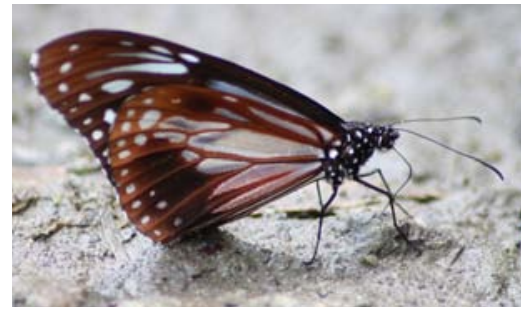

Image 71. Parantica melaneus plateniston

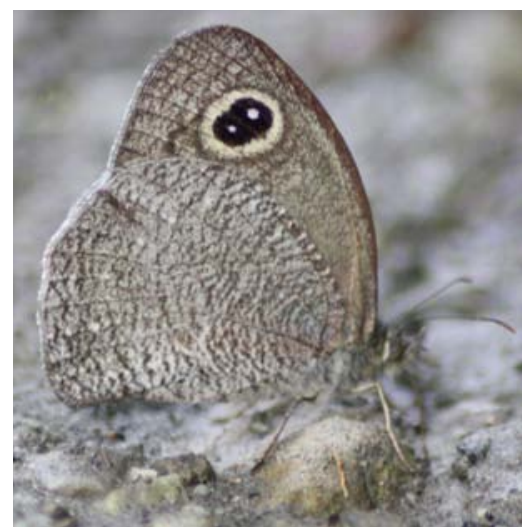

Image 74. Hemadara narasingha

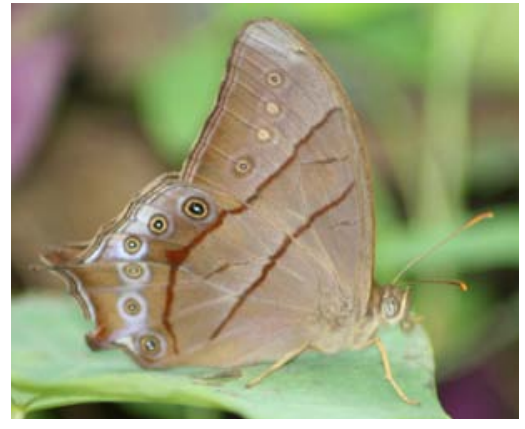

Image 76. Lethe sinorix 


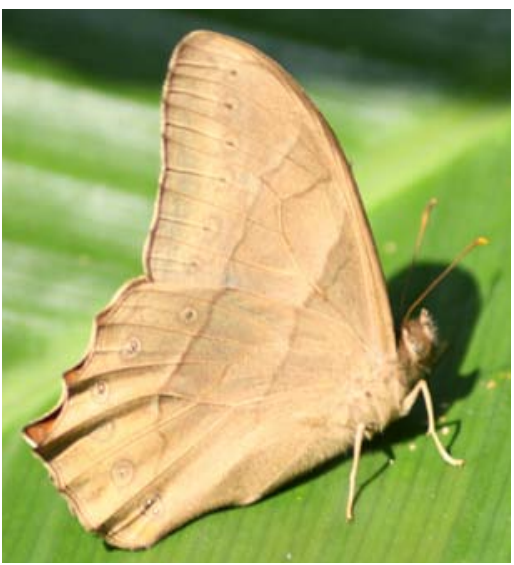

Image 77. Lethe mekara zuchara

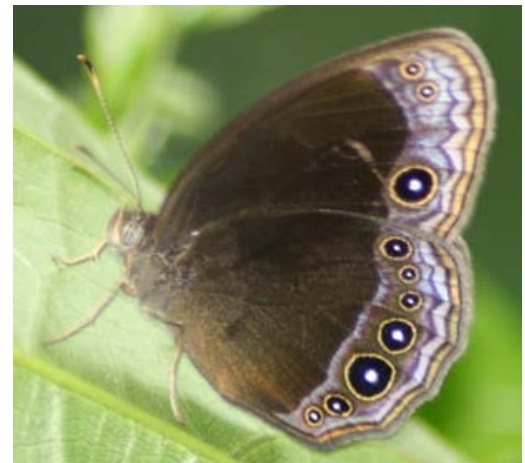

Image 80. Mycalesis adamsoni

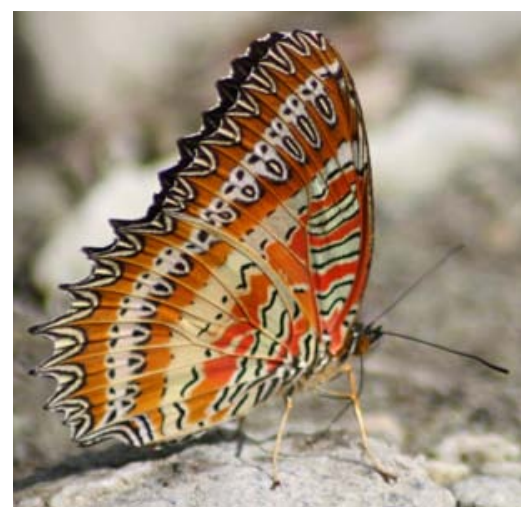

Image 83. Cethosia bibles tisamena

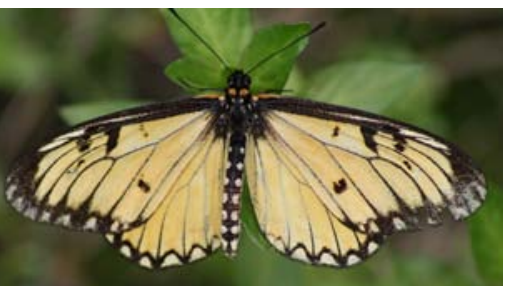

Image 86. Acraea issoria issoria

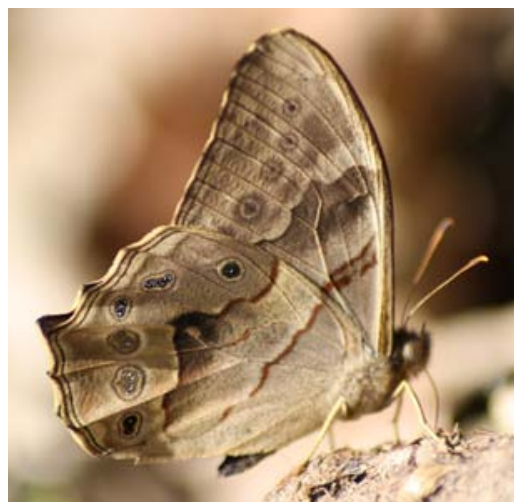

Image 78. Lethe chandica flanona

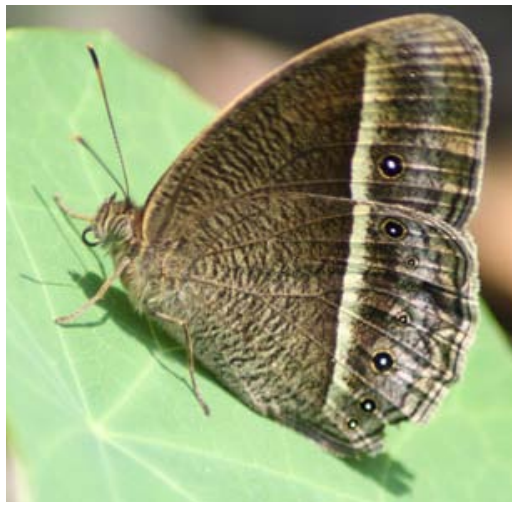

Image 81. Mycalesis misenus misenus

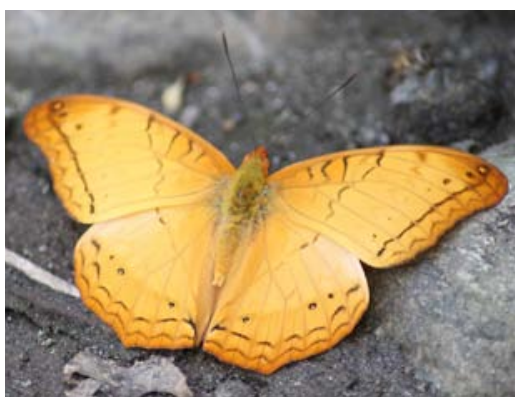

Image 84. Vindula erota erota

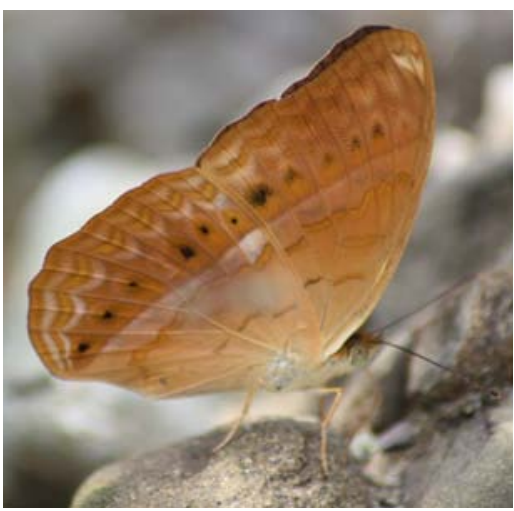

Image 87. Cirrochroa tyche mithila

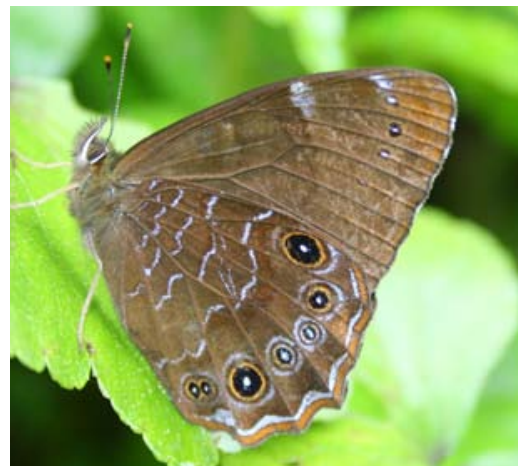

Image 79. Lethe sidonis sidonis

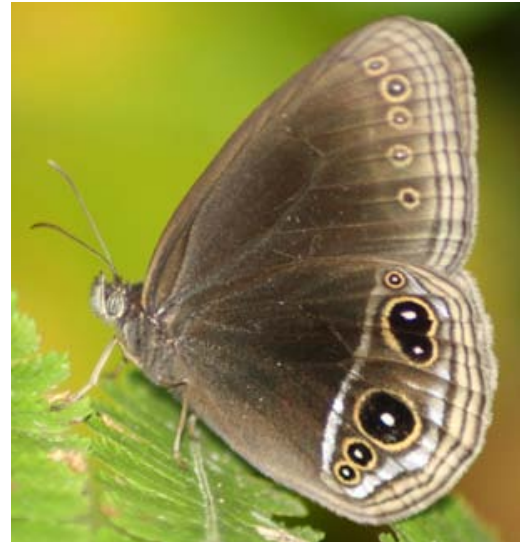

Image 82. Zipoetis scylax

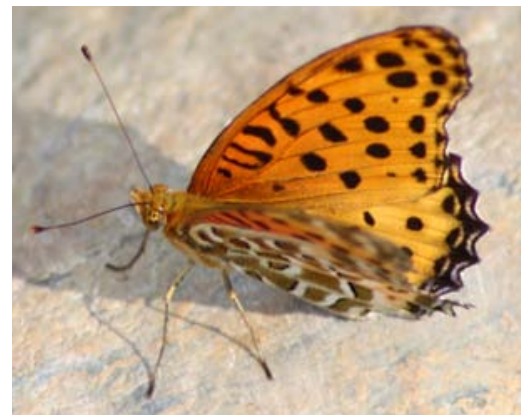

Image 85. Argyreus hyperbius hyperbius

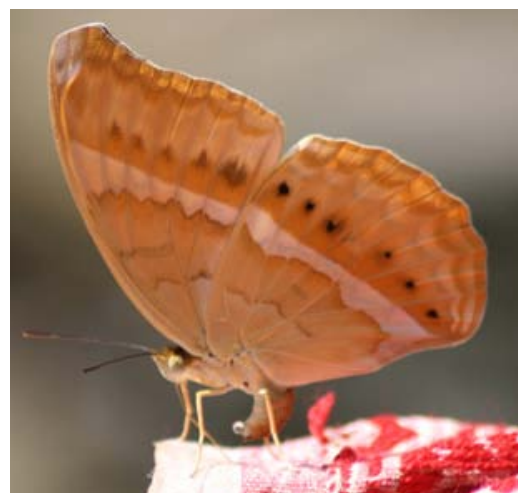

Image 88. Cirrochroa aoris aoris 


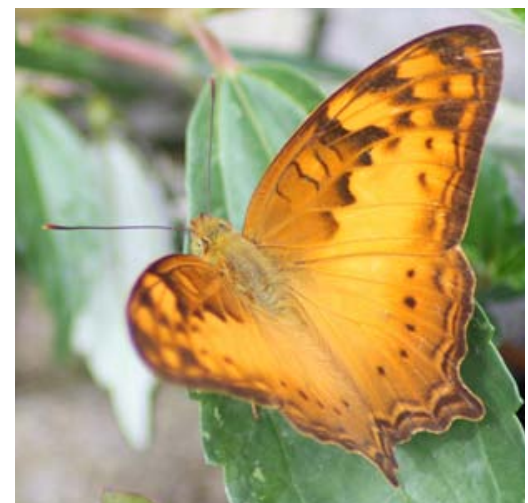

Image 89. Vagrans gista sinha

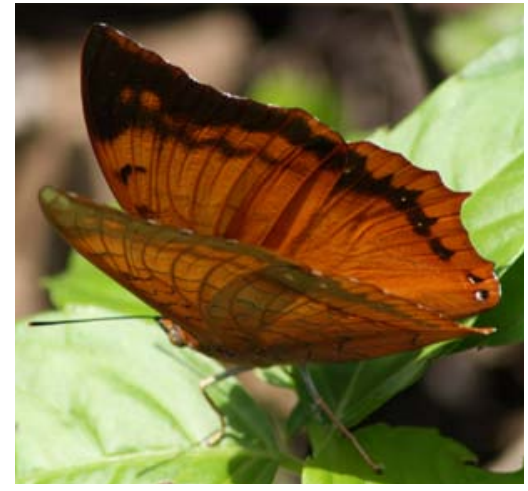

Image 92. Charaxes aristogiton

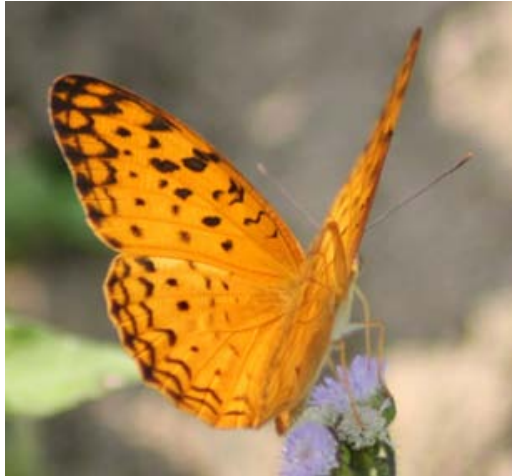

Image 95. Phalanta phalantha phalantha

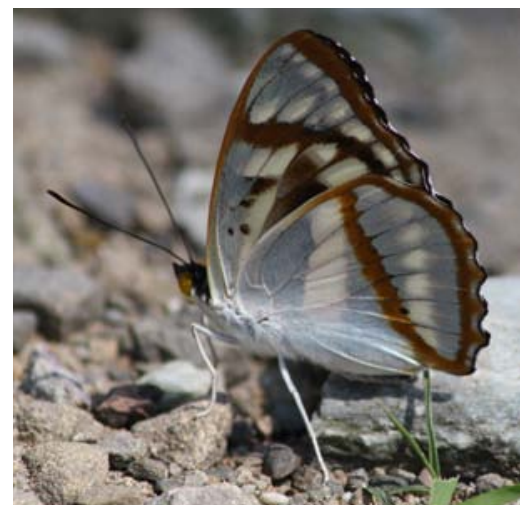

Image 98. Apatura chevana

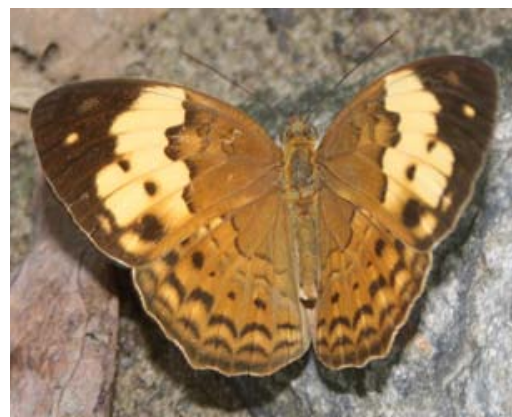

Image 90. Cupha erymanthis lotis

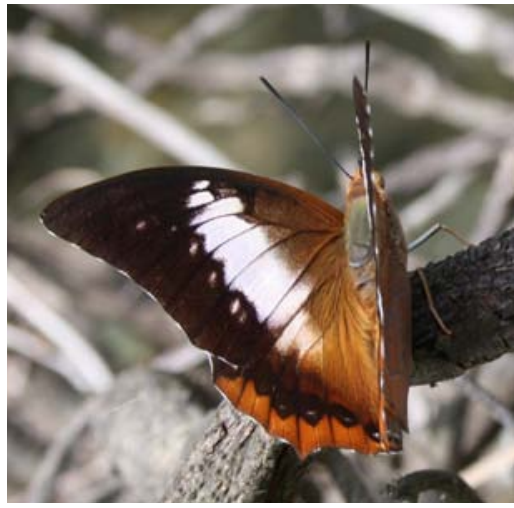

Image 93. Charaxes polyxena hierax

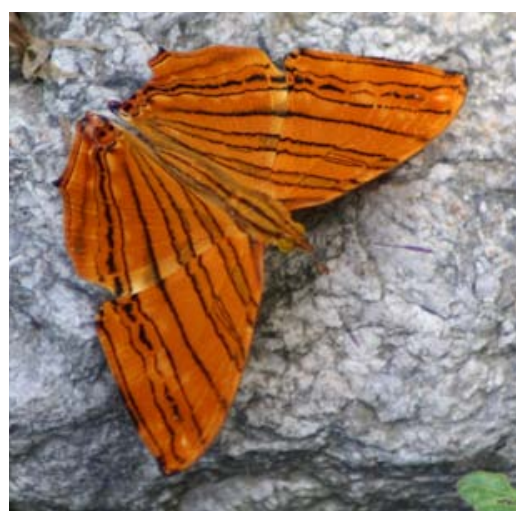

Image 96. Chersonesia risa

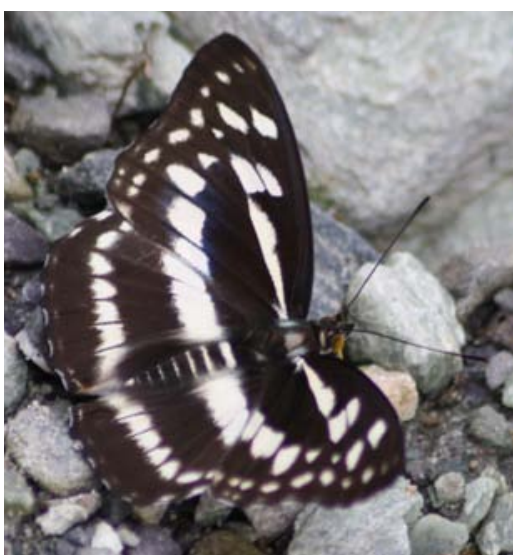

Image 99. Apatura chevana

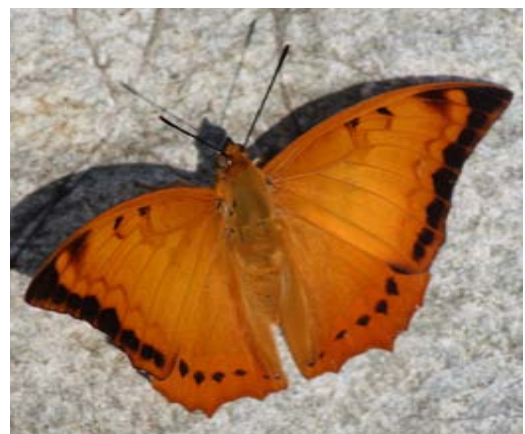

Image 91. Charaxes marmax marmax

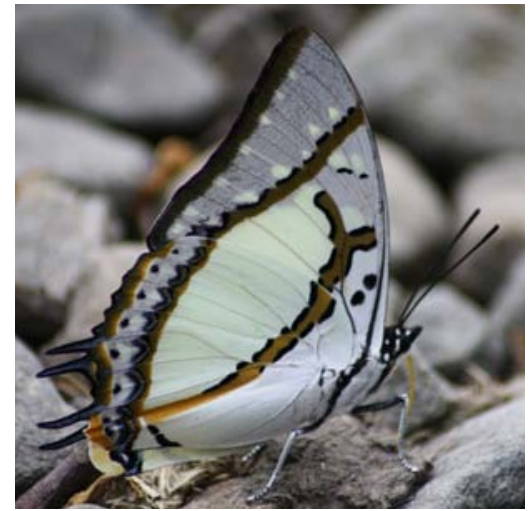

Image 94. Charaxes eudamippus

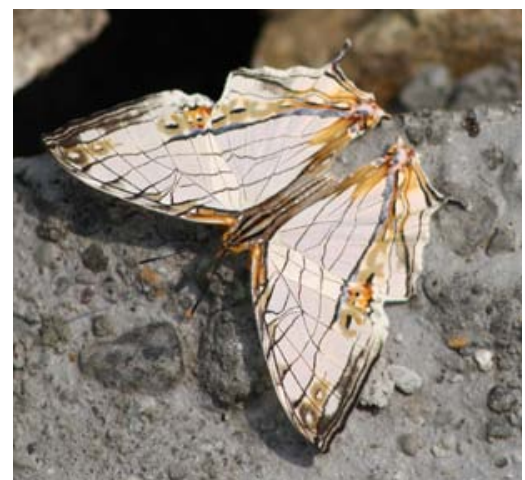

Image 97. Cyrestis thyodamas thyodamas

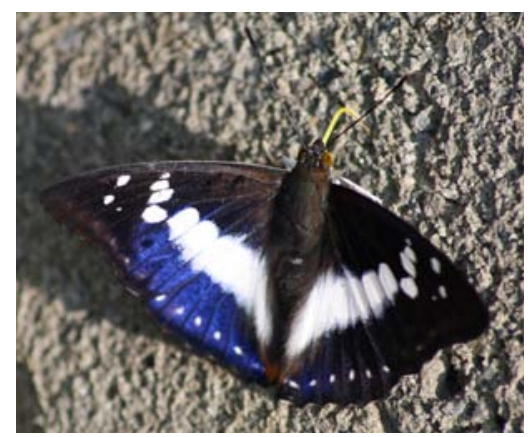

Image 100. Apatura ambica ambica

Journal of Threatened Taxa | www.threatenedtaxa.org | October 2012 | 4(12): 3137-3160 


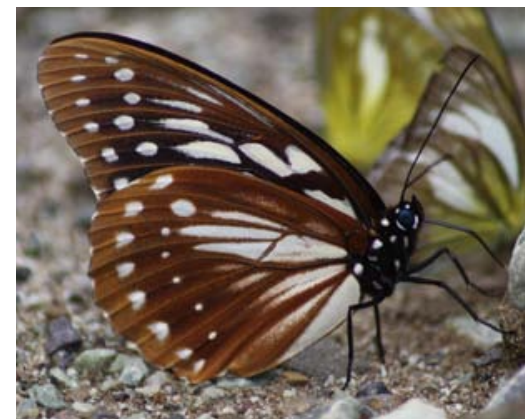

Image 101. Penthema lisarda lisarda

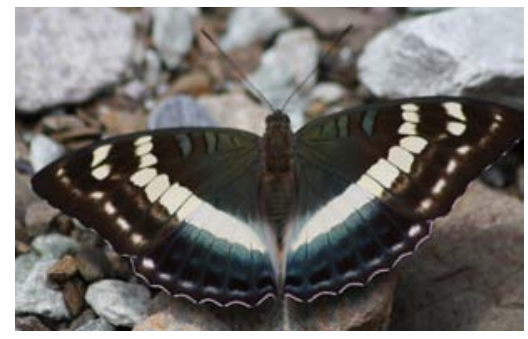

Image 104. Euthalia franciae

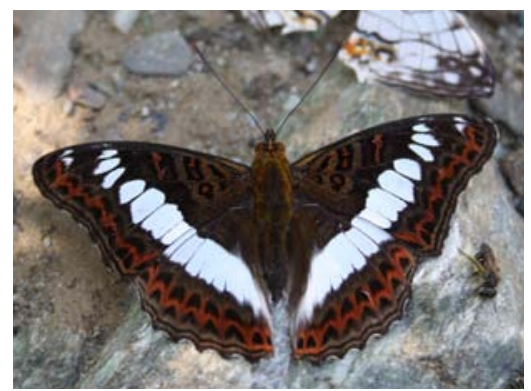

Image 107. Sumalia zulema

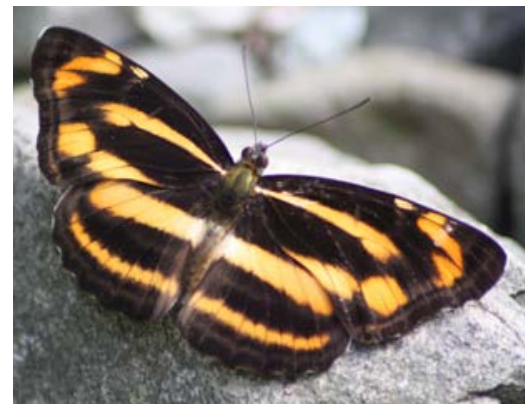

Image 110. Neptis radha radah

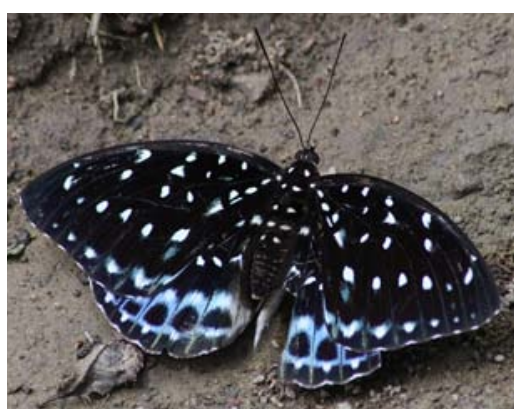

Image 102. Lexias dirtea khasiana

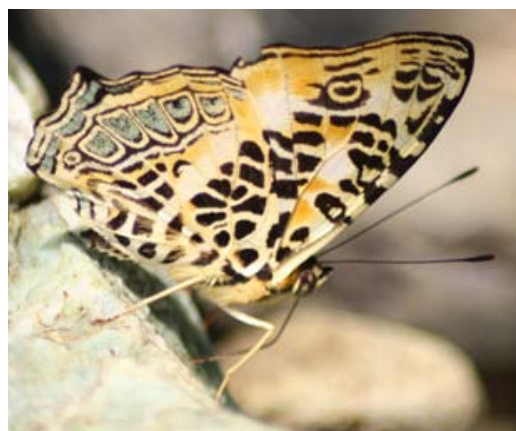

Image 105. Symbrenthia hypselis cotanda

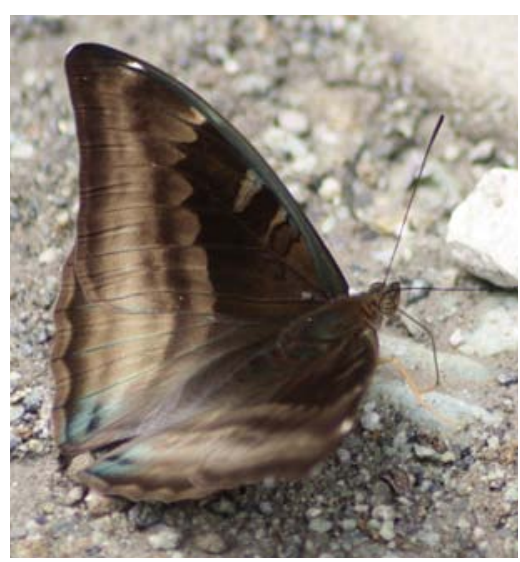

Image 108. Auzakia danava danava

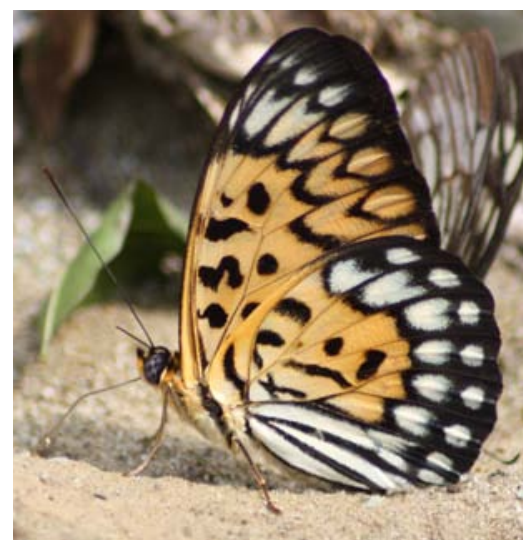

Image 103. Neurosigma doubledayi

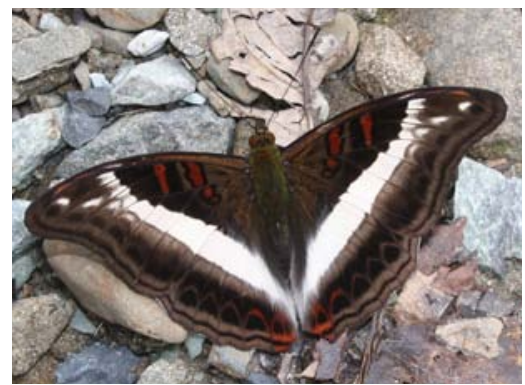

Image 106. Parasarpa dudu dudu

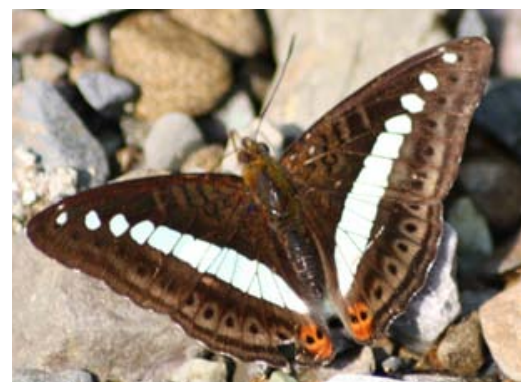

Image 109. Sumalia daraxa daraxa

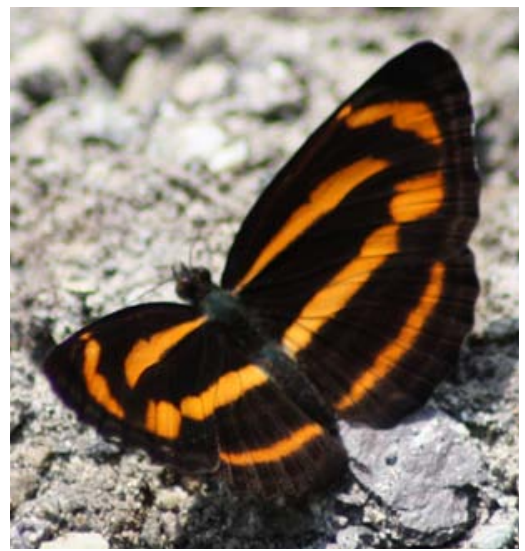

Image 111. Neptis miah miah 


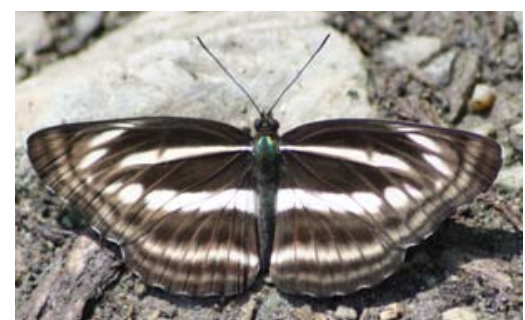

Image 112. Neptis sankara quilta

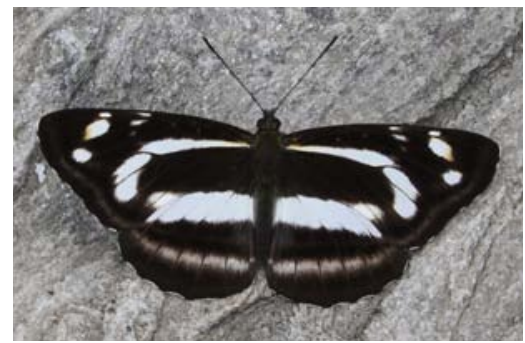

Image 115. Neptis mansara

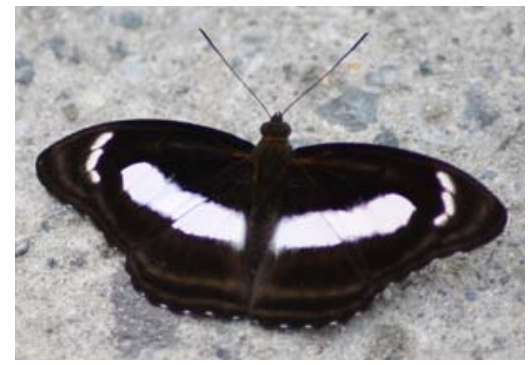

Image 118. Athyma zeroca

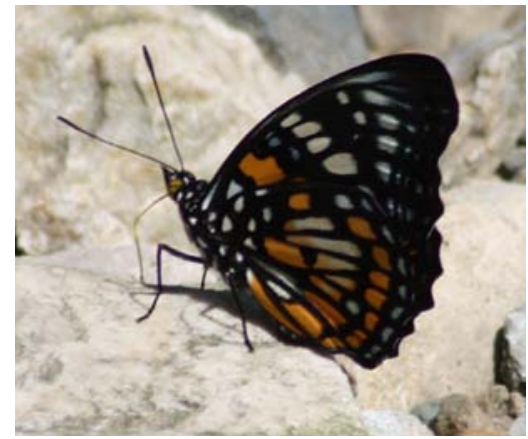

Image 121. Sephisa chandra

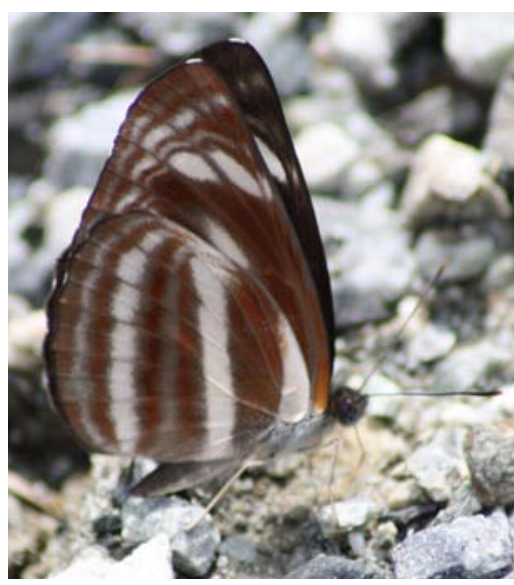

Image 113. Neptis cartica cartica

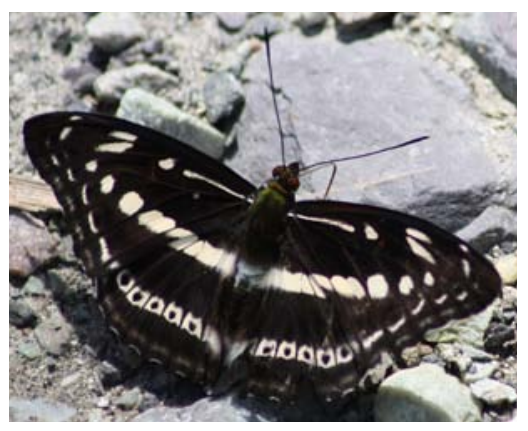

Image 116. Athyma asura asura

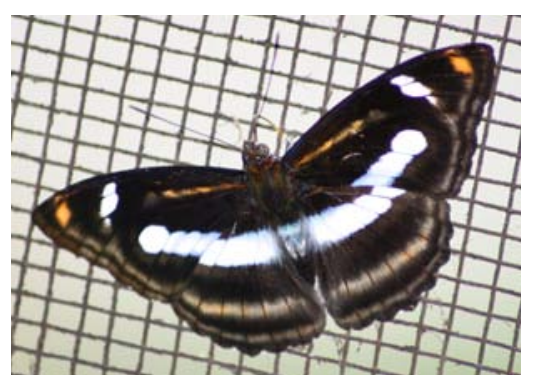

Image 119. Athyma cama

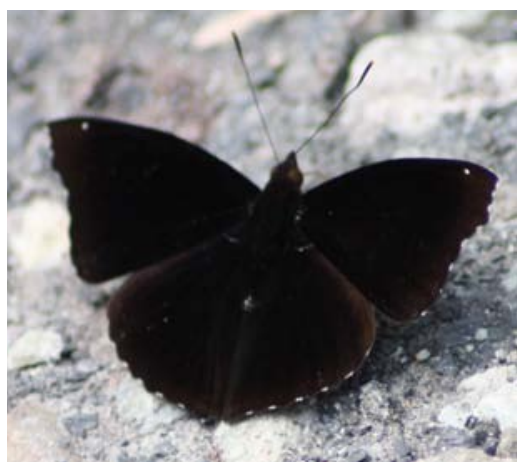

Image 122. Aparuta paristis parisits

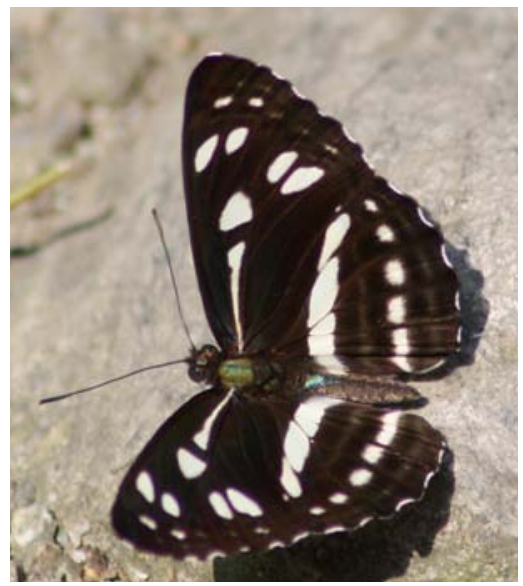

Image 114. Phaedyma columella ophiana

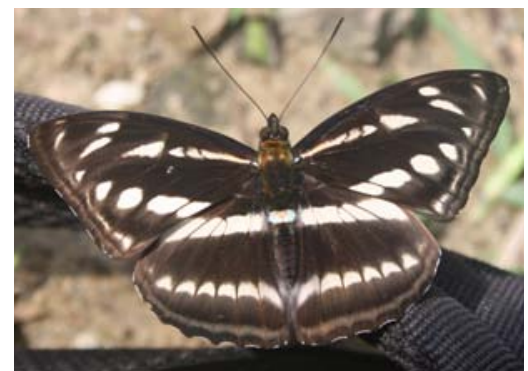

Image 117. Athyma opalina orientalis

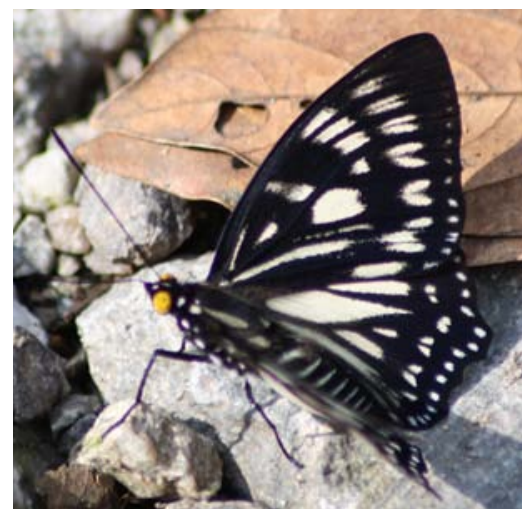

Image 120. Euripus halitherses nyctelius 


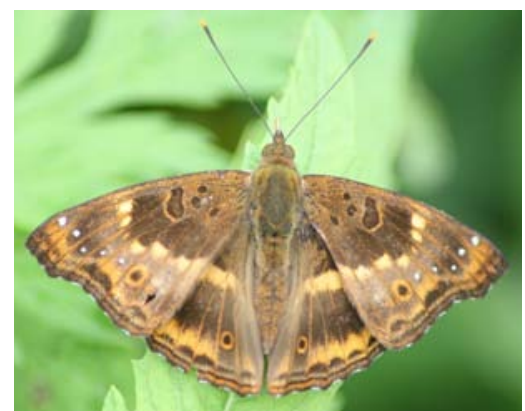

Image 123. Aparuta parvata

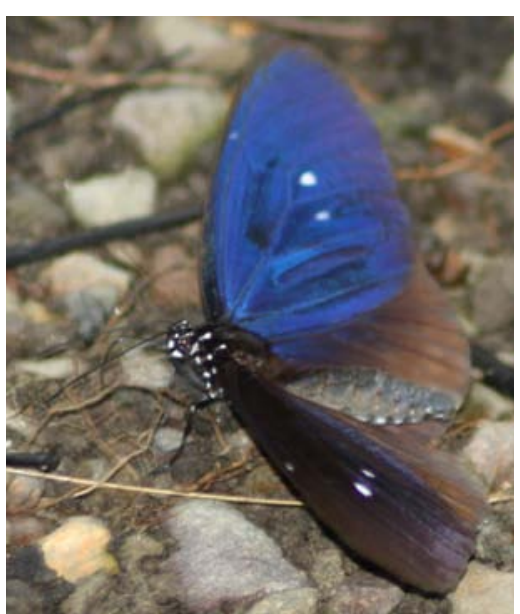

Image 126. Euploea midamus splendens

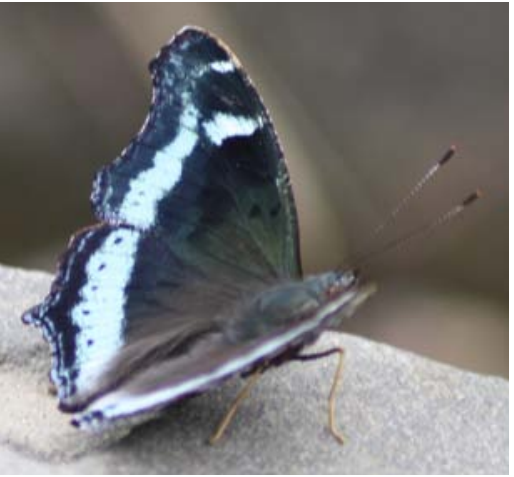

Image 128. Kaniska canace canace
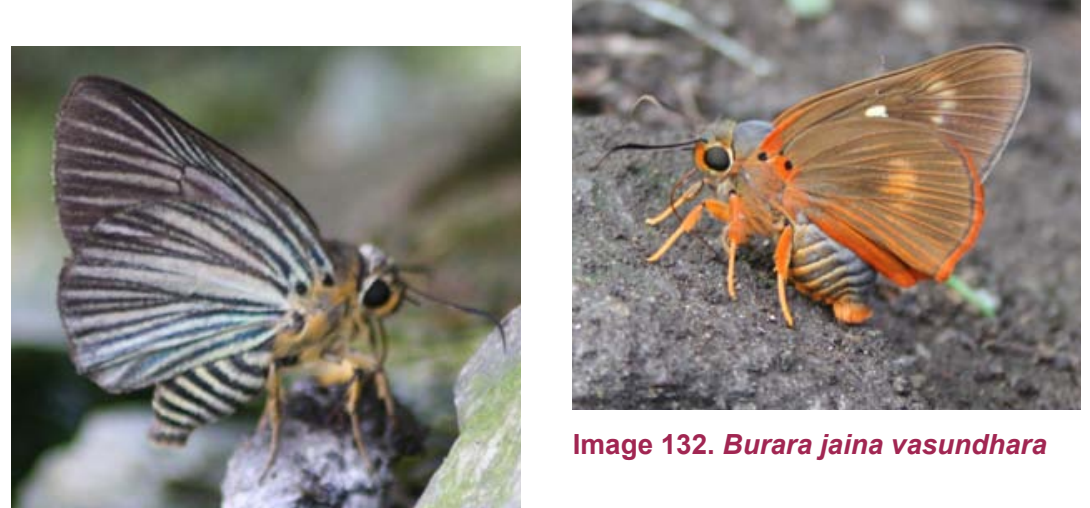

Image 132. Burara jaina vasundhara

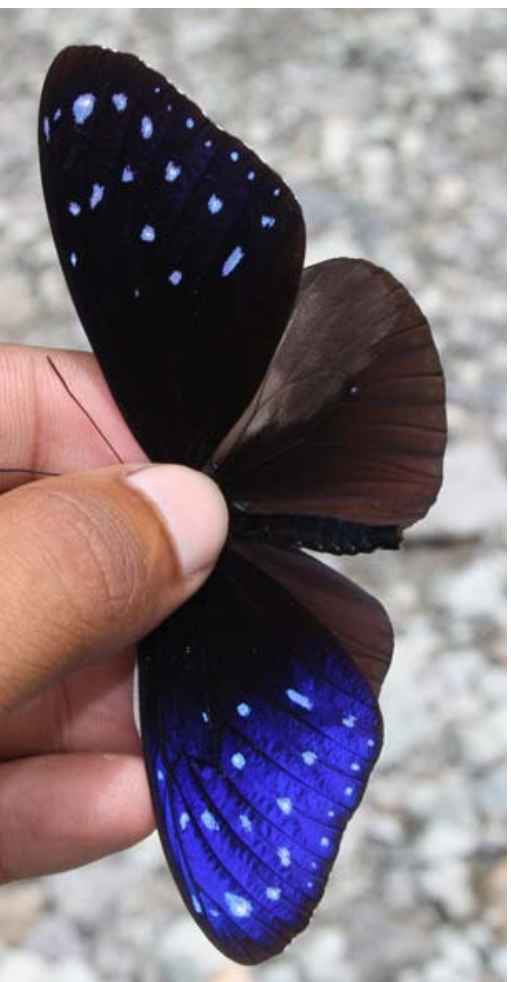

Image 125. Euploea mulciber mulciber

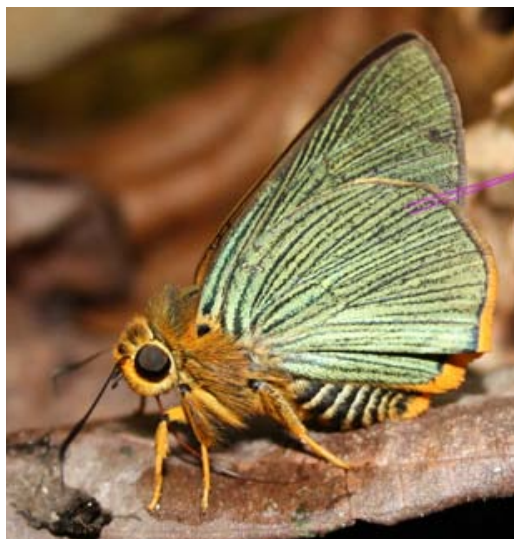

Image 129. Burara vasutana?

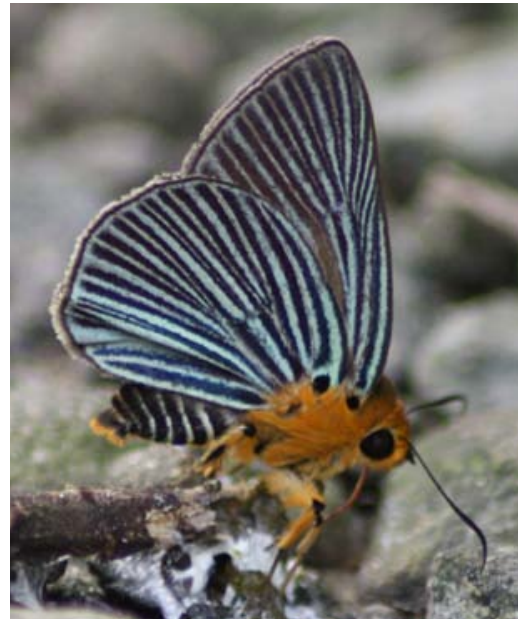

Image 130. Burara amara

Image 131. Burara gomata gomata 


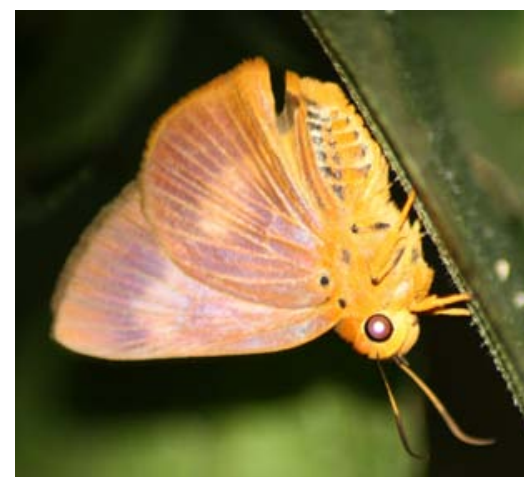

Image 133. Burara oedipodea aegina

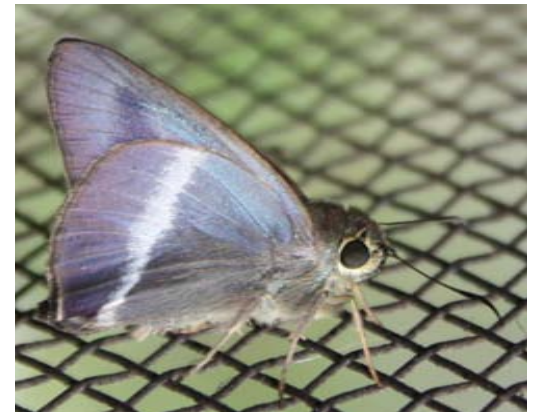

Image 136. Hasora taminatus bhavara

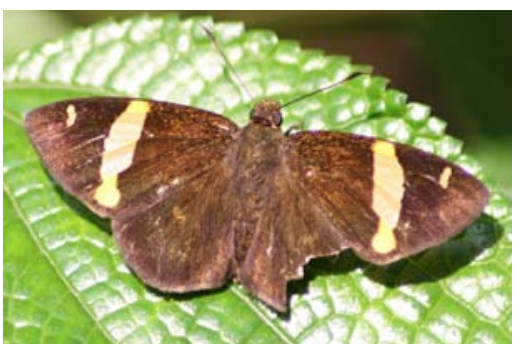

Image 139. Celaenorrhinus aurivittata aurivittata

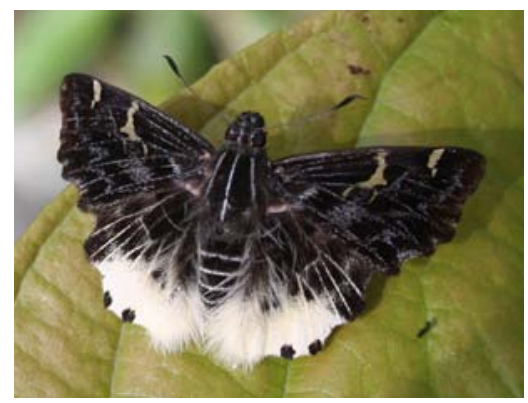

Image 142 . Darpa hanria

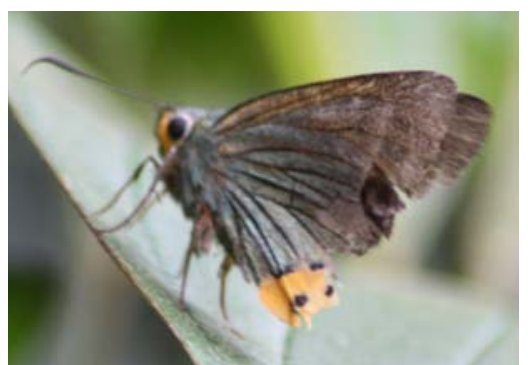

Image 134. Choaspes benjaminii

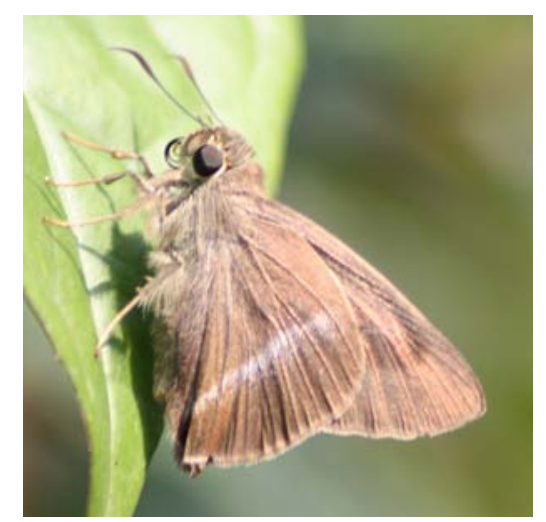

Image 137. Hasora alexis alexis

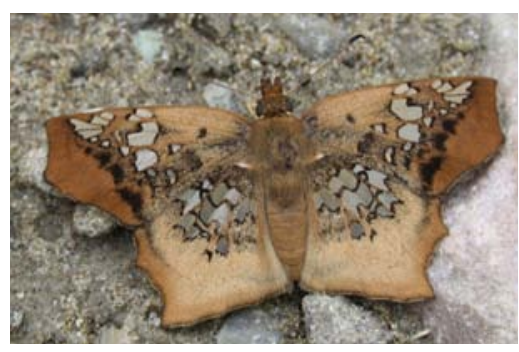

Image 140. .Ctenoptilum vasava

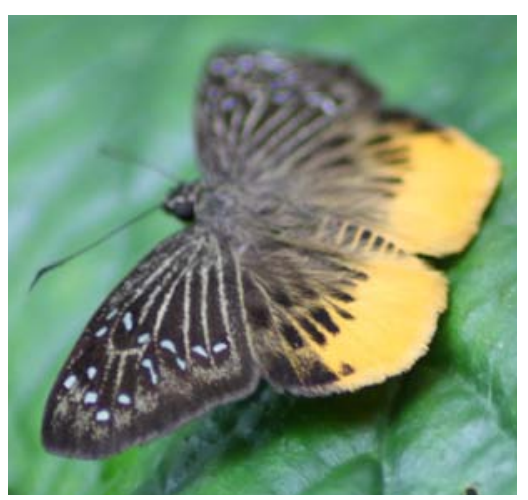

Image 143. Mooreana trichoneura pralaya

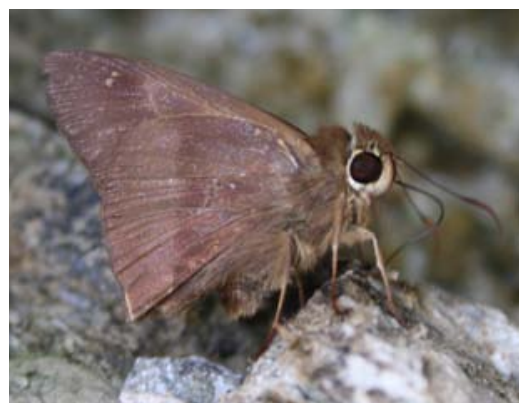

Image 135. Hasora anura danda

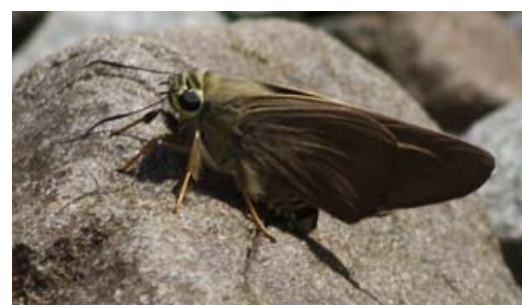

Image 138. Badamia exclamationis

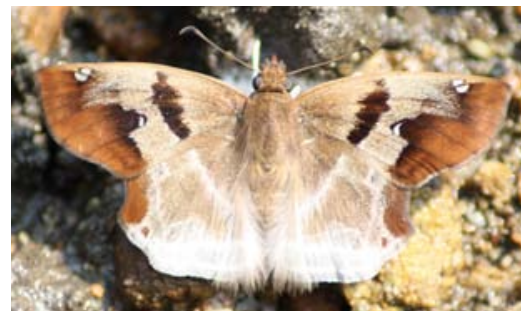

Image 141. Odontoptilum angulata sura

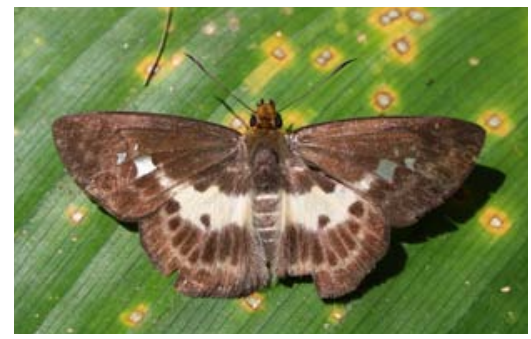

Image 144. Gerosis phisara phisara 


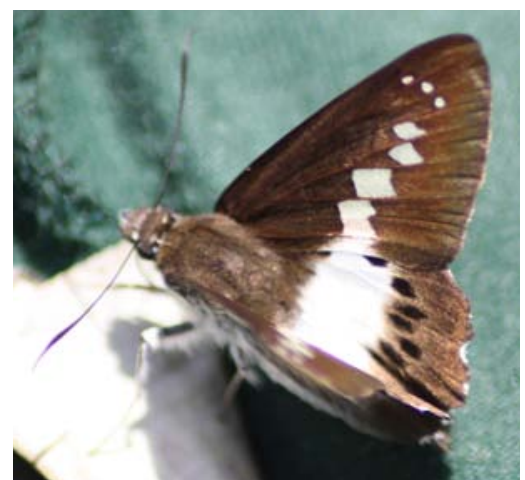

Image 145. Seseria dohertyi

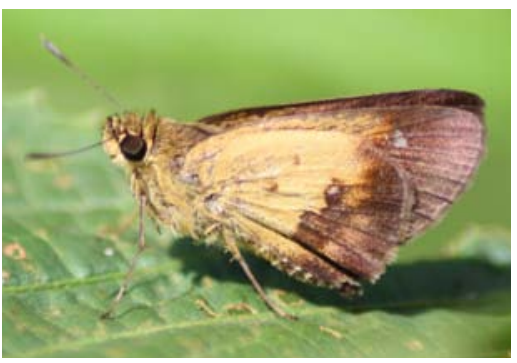

Image 148. Zographetus satwa

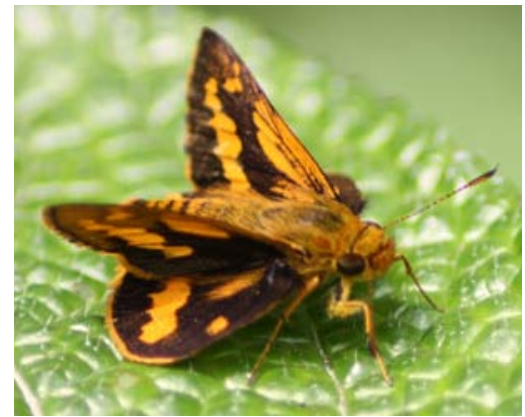

Image 151. Potanthus tropica (male)

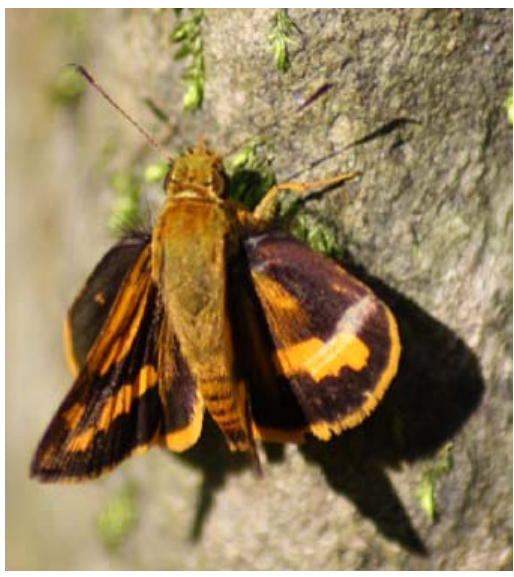

Image 154. Potanthus trachala tytleri (upperside)

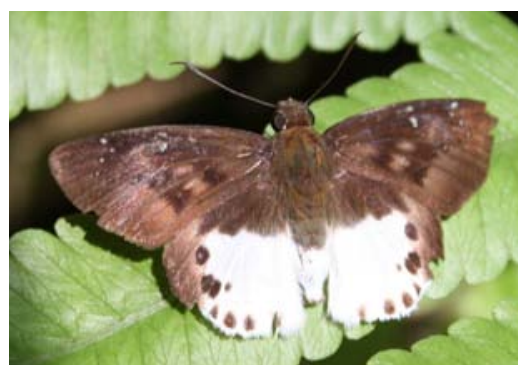

Image 146. Tagiades litigiosa litigiosa

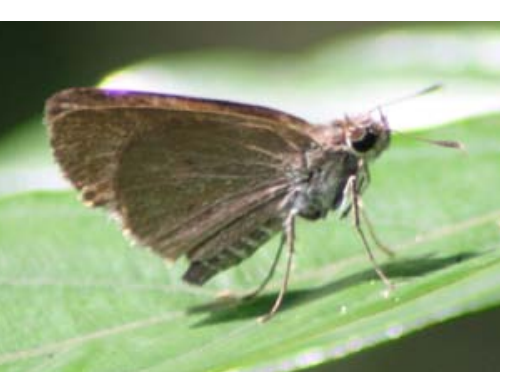

Image 149. Suastus minuta aditia

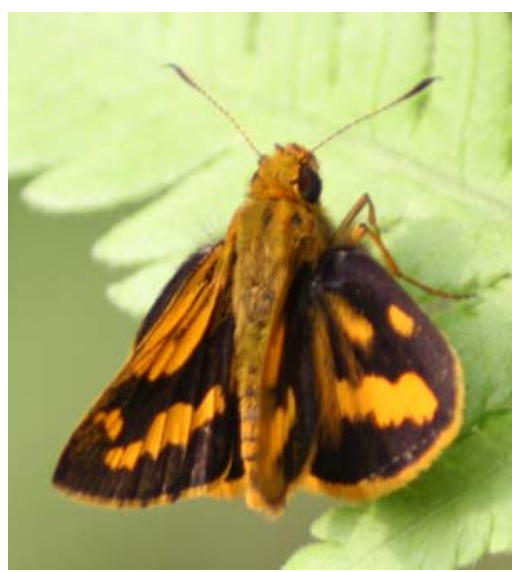

Image 152. Potanthus tropica (female)

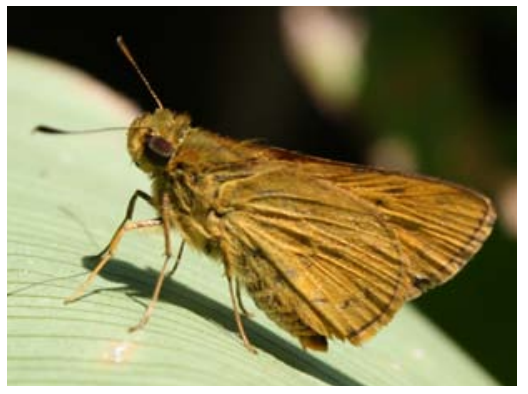

Image 155. Potanthus mara (underside)

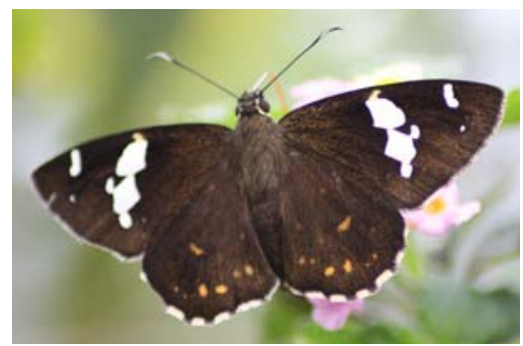

Image 147. Celaenorrhinus leucocera chinensis

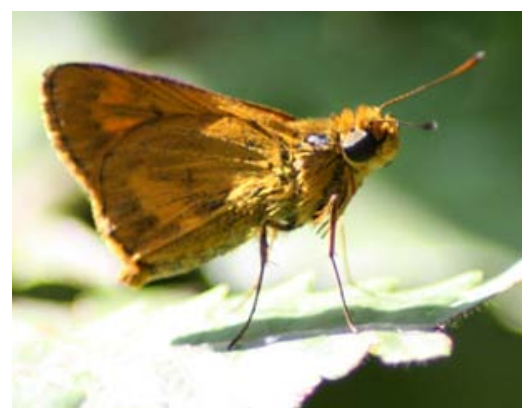

Image 150. Oriens gola gola

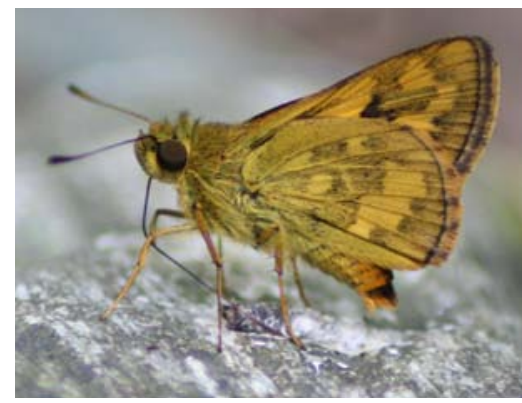

Image 153. Potanthus trachala tytleri (underside)

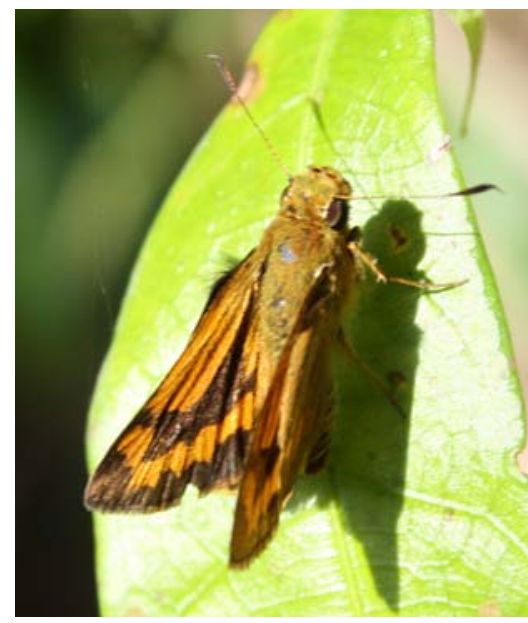

Image 156. Potanthus mara

(upperside) 


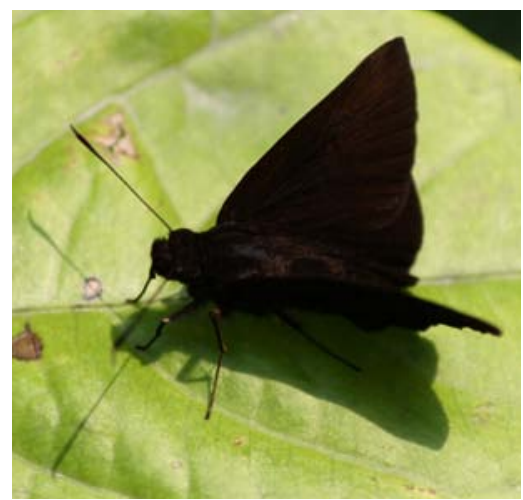

Image 157. Koruthaialos butleri butleri

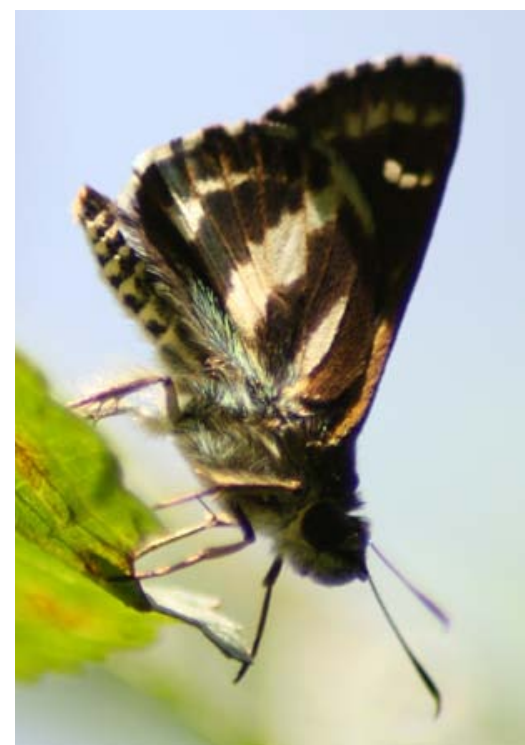

Image 160. Sebastonyma dolopia

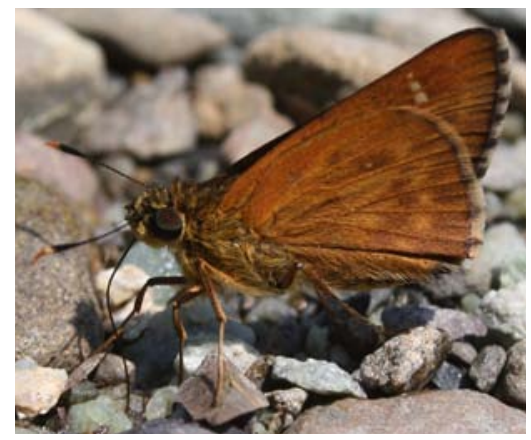

Image 163. Thoressa hyrie (underside)

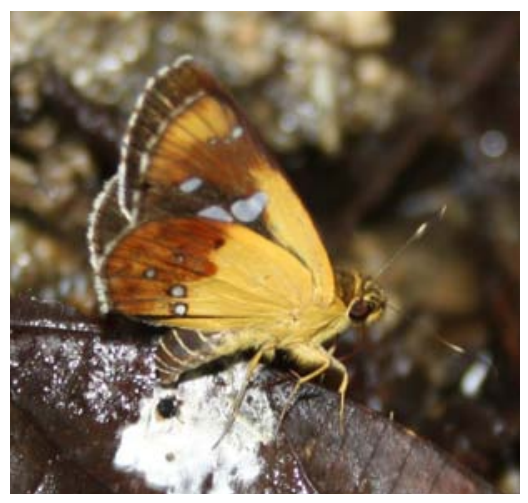

Image 158. Scobura cephaloides cephaloides

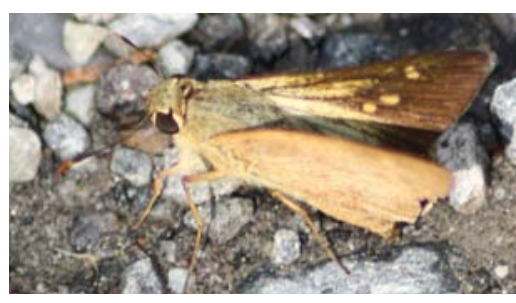

Image 161. Pithauria stramineipennis

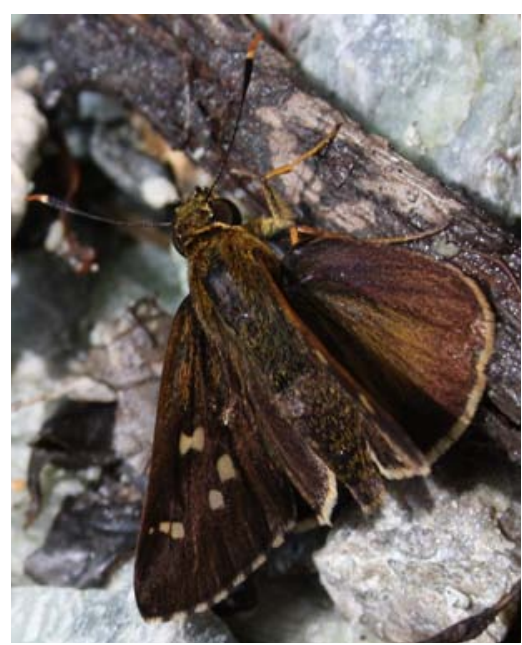

Image 164. Thoressa hyrie (upperside)

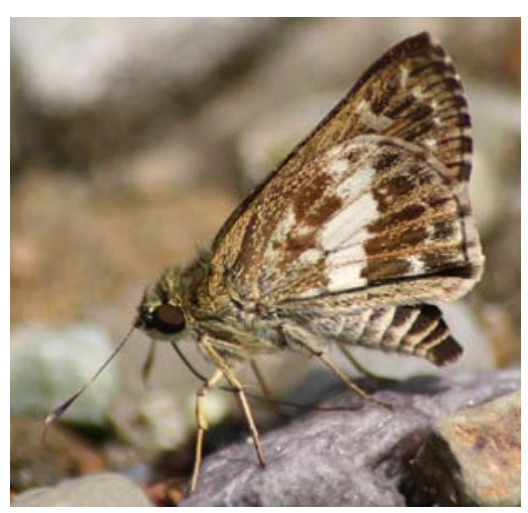

Image 166. Halpe porus

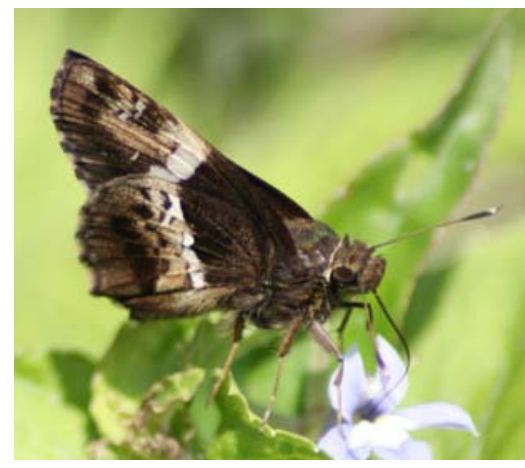

Image 159. Hyarotis adrastus praba

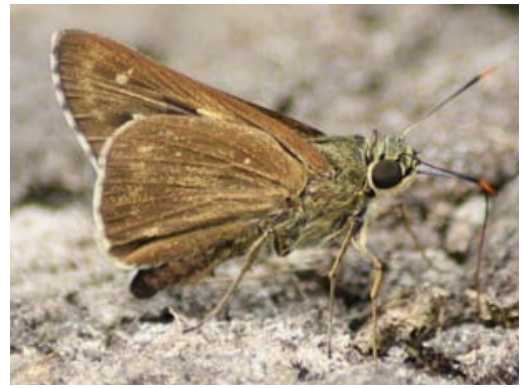

Image 162. Pithauria murdava

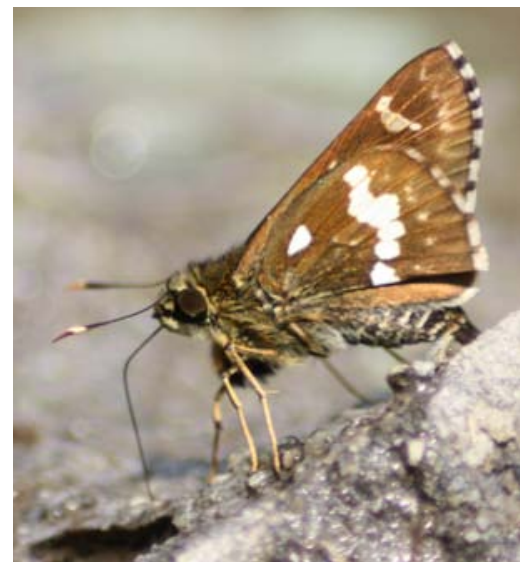

Image 165. Thoressa cerata

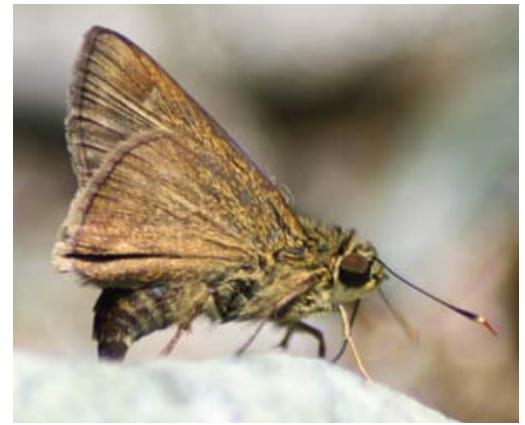

Image 167. Halpe sp. 


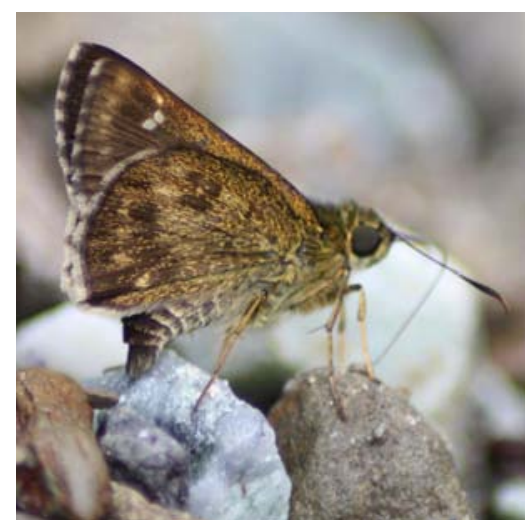

Image 168. Halpe kusala

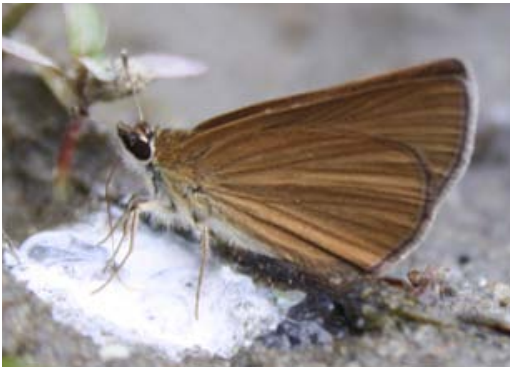

Image 171. Baracus vittatus septentrionum

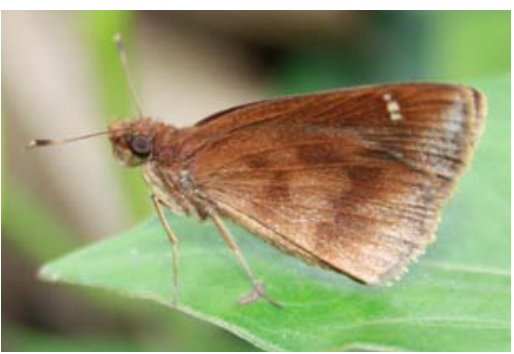

Image 174. Asticopterus jama kada

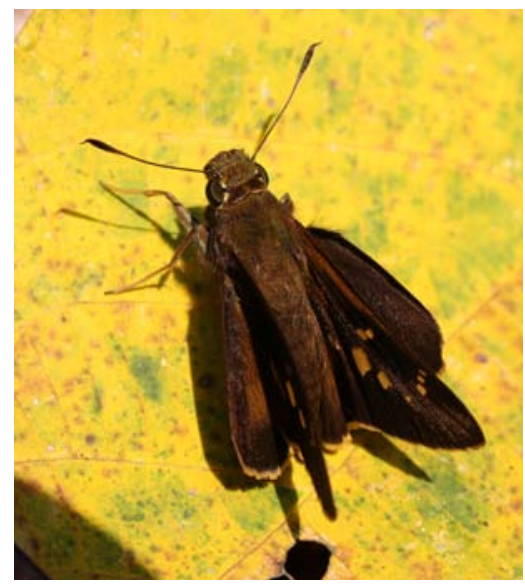

Image 177. Caltoris pagana (upperside)

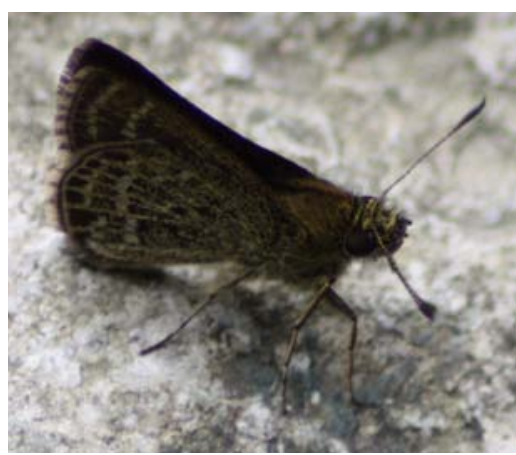

Image 169. Aeromachus stigmata stigmata

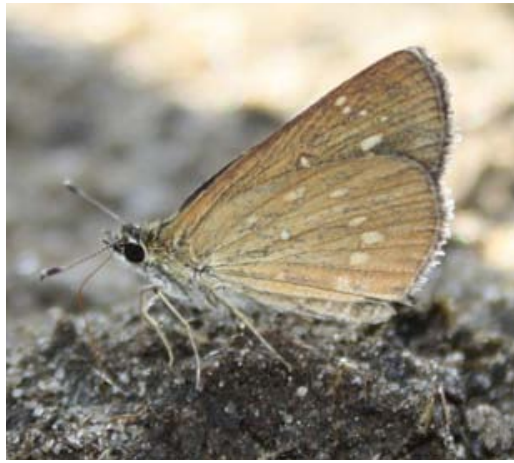

Image 172. Baracus sp.

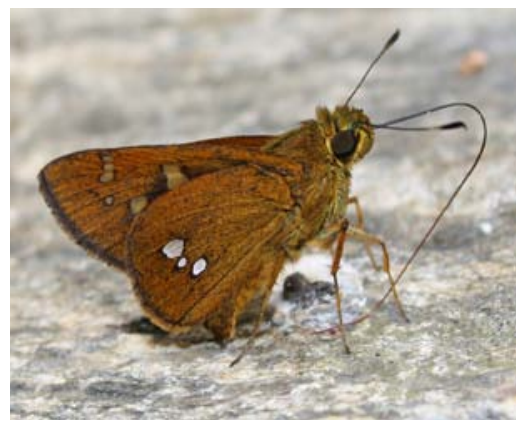

Image 175. Polytremis eltola

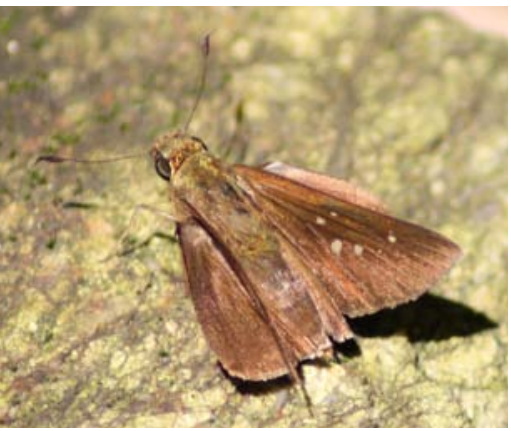

Image 178. Caltoris cahira cara

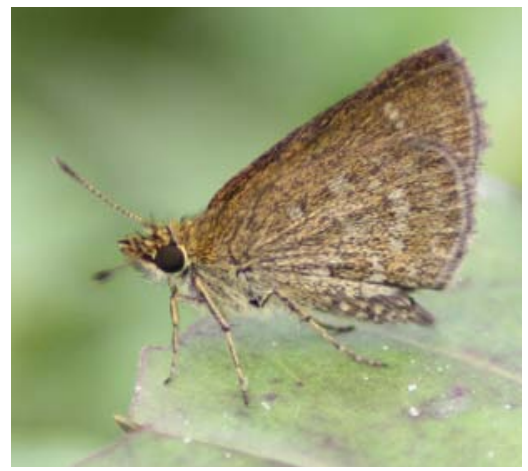

Image 170. Aeromachus jhora creta

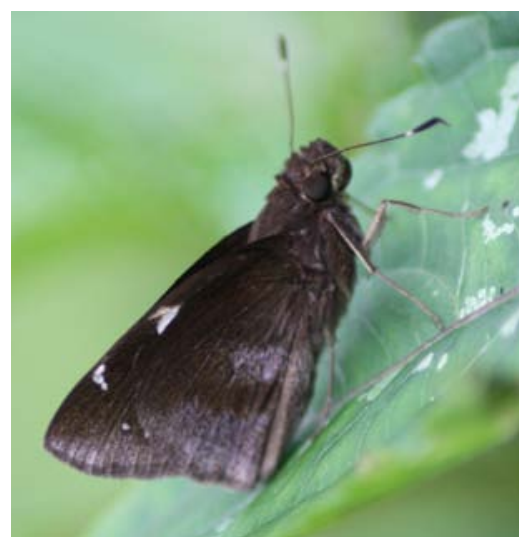

Image 173. Notocrypta feisthameli alysos

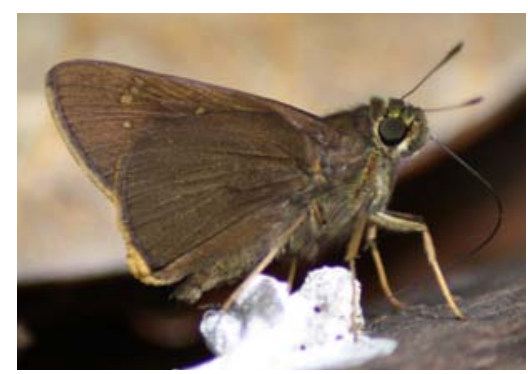

Image 176. Caltoris pagana (underside) 
Butterflies of Dibang Valley

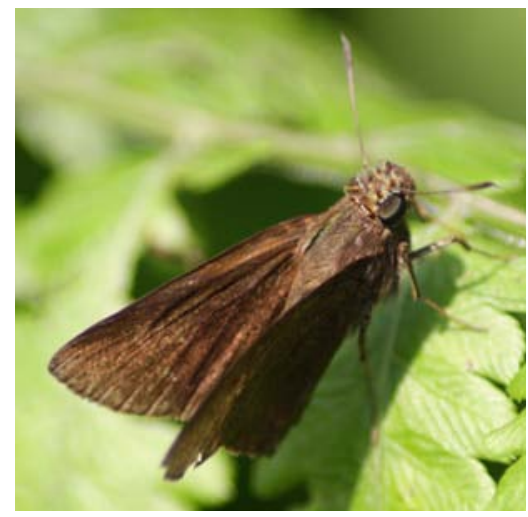

Image 179. Baoris farri

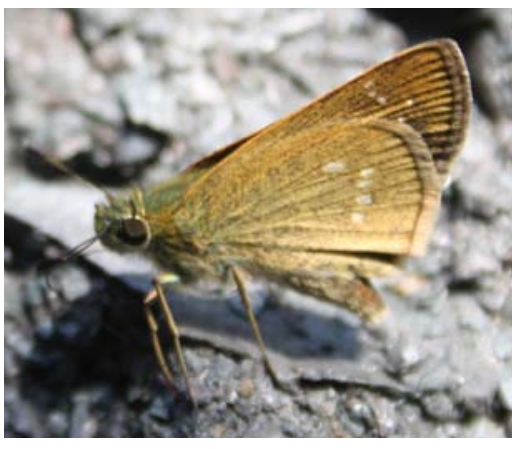

Image 180. Pseudoborbo bevani
M.J.Gogoi

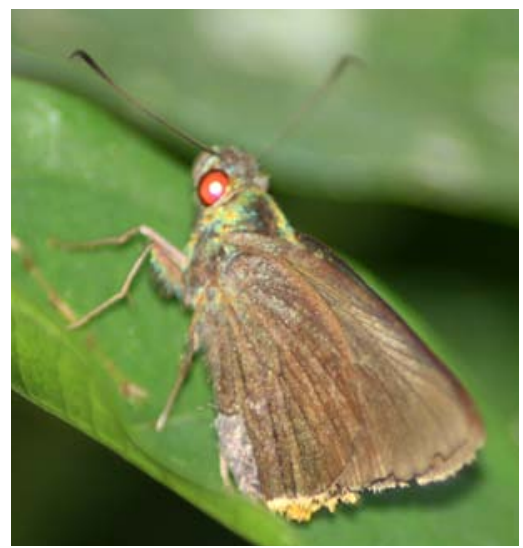

Image 181. Matapa sasivarna 